\title{
COMPLEX STRUCTURES ON REAL PRODUCT BUNDLES WITH APPLICATIONS TO DIFFERENTIAL GEOMETRY
}

\author{
BY \\ RICHARD S. MILLMAN( $\left.{ }^{1}\right)$
}

\begin{abstract}
The purpose of this paper is to classify holomorphic principal fibre bundles which admit a smooth section (i.e. are real product bundles). This is accomplished if the structure group is solvable of type (E). In the general case, a sufficient condition is obtained for a real product bundle to be equivalent to the complex product bundle. A necessary and sufficient condition for the existence of a holomorphic connection on a real product bundle is also obtained. Using this criterion in the case where the structure group is abelian, a generalization of a theorem due to Atiyah (in the case the structure group is $C^{*}$ ) is obtained.
\end{abstract}

\section{Chapter 0: Summary of Main Results}

In his lecture to the International Congress of Mathematicians, 1950, H. Cartan [3] gave a formulation, due to Weil, of the classical second Cousin's problem. The formulation is as follows: Given a holomorphic principal fibre bundle $\xi$ which, when considered as a real $\left(C^{\infty}\right)$ bundle, is the trivial (or product) bundle, how can we decide whether $\xi$ is also trivial as a holomorphic bundle? Rephrasing this slightly, if Pic $(M, G)$ is the set of holomorphic equivalence classes of real trivial holomorphic principal fibre bundles over the complex manifold $M$ with structure group the complex Lie group $G$, how can we describe $\operatorname{Pic}(M, G)$ ? If $G$ is abelian then J.-P. Serre showed that

$$
\operatorname{Pic}(M, G)=\mathscr{D}_{0,1}(M, \hat{G}) / H^{1}(M, \hat{G}), \quad M \text { is compact, }
$$

where $\hat{G}$ is the Lie algebra of $G$, and $\mathscr{D}_{0,1}$ denotes the Dolbeault $\bar{\partial}$ cohomology of $M$. In 1957, J. Frenkel [6] proved that if $G$ is solvable and $\mathscr{D}_{0,1}(M, C)=0$ then Pic $(M, G)=0$. Both Serre's and Frenkel's results use the machinery of sheaftheoretic cohomology. Our solution to this problem lies in a set $\operatorname{Exp} D(M, G)$ and a map $\Xi: \operatorname{Exp} D(M, G) \rightarrow \operatorname{Pic}(M, G)$ which is always onto and is an isomorphism almost every time that $G$ is solvable.

Presented to the Society, January 22, 1971 under the title Real trivial holomorphic principal fibre bundles; received by the editors March 22, 1971.

AMS 1970 subject classifications. Primary 32L05; Secondary 32C10, 53C15, 53C55, 53C05.

Key words and phrases. Holomorphic principal fibre bundle, complex structure, connection, Kähler manifold, complex manifold.

( ${ }^{1}$ ) This paper contains portions of the author's doctoral thesis written at Cornell University. The author would like to express his grateful appreciation to his advisor, Professor H. C. Wang, and to Professor C. J. Earle for their many valuable conversations.

Copyright (c) 1972, American Mathematical Society 
We begin by giving some results of a general nature in the first chapter and then applying them to the above case in the second chapter. (We shall number the results as in this paper.)

Let $M$ (resp. $G$ ) be a connected complex manifold (resp. complex Lie Group). Let $\hat{G}$ be the Lie algebra of $G, \Lambda^{0,1}(M, \hat{G})=\hat{G}$-valued 1 -forms on $M$ of type $(0,1)$. If $P$ is an almost complex manifold with almost complex structure $J_{0}$ then $\xi_{0}: G \rightarrow P \stackrel{\pi}{\rightarrow} M$ is called an almost holomorphic principal fibre bundle (a.h.p.f.b.) if $G$ acts on $P$ in such a way that $\Phi: P \times G \rightarrow P \times P$ is an almost holomorphic diffeomorphism $(p, g) m\left(p, p^{g}\right)$ of $P \times G$ onto a closed subset of $P \times P$ and $M=P / G$ (via $\pi)$. The almost complex structure $J$ on $P$ is said to be coherent with $\xi_{0}$ if $\xi: G \rightarrow P_{J} \stackrel{\pi}{\rightarrow} M$ is an a.h.p.f.b. Let $\operatorname{Ker} \pi$ denote the sub-bundle of $T(P)$ given by $\operatorname{Ker} \pi=\left\{(p, X) \mid X \in T_{p} P, p \in P\right.$ and $\left.\dot{\pi}(X)=0\right\}$.

THEOREM 1.2.1. If $\xi_{0}: G \rightarrow P_{J_{0}} \stackrel{\pi}{\rightarrow} M$ is an a.h.p.f.b. then there is a 1-1 correspondence between almost complex structures on $P$ coherent with $\xi_{0}$ and $\hat{G}$-valued $(0,1)$ forms $\omega$ (with respect to $J_{0}$-structure) on $P$ such that

(1) $\omega(\operatorname{Ker} \pi)=0$,

(2) $\omega$ is of adjoint type.

In fact, $\omega$ is defined by $\left(J-J_{0}\right)_{p}(X)=\theta_{p}\left(\omega_{p}(X)\right)$ where $\theta_{p}$ is the canonical embedding of $\hat{G}$ onto $(\operatorname{Ker} \pi)_{p} \subseteq T_{p}(P)$ for each $p \in P$.

THEOREM 1.2.5. Let $\xi_{0}$ be a holomorphic principal fibre bundle. Let $J_{1}$ be an almost complex structure coherent with $J_{0}$. If $J_{1}$ corresponds to $\omega \in \Lambda^{0,1}(P, \hat{G})$ then $J_{1}$ is a complex structure if and only if the sum of the $(2,0)$ and $(0,2)$ components (with respect to $J_{1}$ ) of $d \omega$ is $-(i / 4)[\omega, \omega]$. In particular, if $J$ is complex then $\bar{\partial}_{J_{1}} \omega$ $=-(i / 4)[\omega, \omega]$.

THEOREM 1.2.6. There is a 1-1 correspondence between $\Lambda^{0,1}(M, \hat{G})$ and almost complex structures on $M \times G$ coherent with the product structure. In fact, this correspondence is given by: for each $\eta \in \Lambda^{0,1}(M, \hat{G}), \eta \leadsto J^{\eta}$ where

$$
J_{z, \lambda}^{\eta}(W, Z)=\left(J_{M} W, J_{G} Z+\left(d R_{\lambda}\right)_{e} \eta(W)\right)
$$

for $z \in M, \lambda \in G, W \in T_{z}(M), Z \in T_{\lambda}(G)$.

Let $\sim_{\exp }$ be the equivalence relation generated by: for $\omega, \eta \in \Lambda^{0,1}(M, \hat{G})$, $\omega \sim \exp \eta \Leftrightarrow \exists h: M \rightarrow \hat{G}$ such that for all $A \in T_{z}(M)$

$$
2 i\left(\left(e^{-\mathrm{ad} h(z)}-I\right) / \mathrm{ad} h(z)\right)(\bar{\partial} h(A))=\omega(A)-e^{-\mathrm{ad} h(z)} \eta(A) .
$$

$\omega$ is expexact if $\omega \sim \exp 0$. Let $Z \exp D(M, \hat{G})$ be the set of all $(0,1)$ forms $\omega$ such that $\omega$ is locally expexact. Let $\operatorname{Exp} D(M, G)=Z \exp D(M, \hat{G}) / \sim_{\text {exp }}$. We say that $M$ have the exponential lift property with respect to $G$ if any map $s: M \rightarrow G$ can be factored through exp: $\hat{G} \rightarrow G$. 
THEOREM 2.2.4. (a) There is a surjection for any $M$ and $G$,

$$
\Xi: \operatorname{Exp} D(M, G) \rightarrow \operatorname{Pic}(M, G)
$$

given by $\Xi(\eta)=\left\{J^{\eta}\right\}$.

(b) If $M$ has exponential lift with respect to $G$ then $\Xi$ is an isomorphism.

In $\S 3$ of Chapter II, we compute $\operatorname{Exp} D$ under certain circumstances and so are able to prove Serre's, Frenkel's and a converse of Frenkel's result; to wit

Theorem 2.3.1. If $G$ is abelian then $\operatorname{Exp} D(M, G)=\mathscr{D}_{0,1}(M, \hat{G})$.

Theorem 2.3.2. If $[\hat{G}, \hat{G}] \neq \hat{G}$ then $\operatorname{Exp} D(M, G)=0$ implies $\mathscr{D}_{0,1}(M, C)=0$.

THEOREM 2.3.4. If $G$ is solvable then $\mathscr{D}_{0,1}(M, C)=0$ implies $\operatorname{Exp} D(M, G)=0$.

THEOREM 2.3.5. Let $\eta \in \Lambda^{0,1}(M, \hat{G})$ correspond to $J^{\eta}$ as in Theorem 1.2.5. Then the following are equivalent:

(a) $\eta \in Z \exp D(M, G)$,

(b) $J^{\eta}$ is a complex structure,

(c) $\bar{\partial} \eta=(i / 4)[\eta, \eta]$.

In Chapter III, we first apply the above to the theory of holomorphic connections obtaining the following necessary and sufficient condition for the existence of a holomorphic connection on a real product bundle:

THEOREM 3.1.5. The set of holomorphic connections on $G \rightarrow(M \times G)_{J^{n}} \rightarrow M$ is in 1-1 correspondence with the set of all $\beta \in \Lambda^{1,0}(M, \hat{G})$ such that $\bar{\partial} \beta=\partial \eta-(i / 2)[\eta, \beta]$.

THeOREM 3.1.7. If $G$ is abelian, $M$ compact Kähler, then $G \rightarrow(M \times G)_{J^{\omega}} \rightarrow M$ possesses a holomorphic connection.

The above theorem and also its converse were first proved by Atiyah [1] in the case when $G=C^{*}$. We prove the converse in the abelian case under the further assumption that $M$ is simply connected.

In the last section we put a natural Hermitian metric on $(M \times G)_{J^{n}}$ and show that if $G$ is a complex torus then $(M \times G)_{J^{\eta}}$ is Kähler in this metric if and only if $d \eta=0$.

Chapter I: Almost Holomorphic Principal Fibre Bundles

1. Preliminaries. We shall first review some of the basic definitions about complex manifolds, mostly to fix terminology. We shall follow [10] as a standard reference. Throughout this work, a manifold (resp. Lie group) will always mean a connected, paracompact and Hausdorff manifold (resp. Lie group). We write $\hat{G}$ for the Lie algebra of the Lie group $G$. $\hat{G}$ is, in particular, a complex vector space with $\operatorname{dim}_{C} \hat{G}=\operatorname{dim}_{C} G$. Let $M$ be a real manifold, $T_{R}(M)$ its (real) tangent bundle and $T(M)=T_{R}(M) \otimes C$ be the complex tangent bundle of $M$, i.e., $T(M)$ $=\left\{X+i Y \mid X, Y \in T_{R}(M)\right\}$. In the future by tangent vector, 1-form, etc. we mean complex tangent vector, 1 -form, etc. Let $J$ be an almost complex structure on $M$ 
(i.e., an endomorphism of $T_{R}(M)$ such that $J^{2}=-I$ ). We shall occasionally write $M_{J}$ to mean the almost complex manifold $M$ with structure $J$. If $\psi: M \rightarrow N$ we shall write $d \psi_{z}$ or $\psi_{z}$ for the differential of $\psi$ at the point $z \in M$; that is $d \psi_{z}$ $=\psi_{z}: T_{z}(M) \rightarrow T_{\psi(z)} N$. Let $V$ be a complex vector space and let $\Lambda^{p}(M, V)$ $=\Lambda^{p}\left(\operatorname{Hom}_{C}(T(M), V)\right)$ be the complex vector space of $V$-valued $p$-forms on $M$ and $\Lambda(M, V)=\oplus \Lambda^{p}(M, V)$. If

$$
T^{1,0}(M)=\{X \in T(M) \mid J X=i X\} \quad \text { and } \quad T^{0,1}(M)=\{X \in T(M) \mid J X=-i X\},
$$

then the eigenspace decomposition of $T(M)=T^{1,0}(M) \oplus T^{0,1}(M)$ induces a decomposition $\Lambda(M, V)=\oplus \Lambda^{p, q}(M, V)$. An element $\omega \in \Lambda^{p, q}(M, V)$ is called a form of type $(p, q)$. In particular, $\omega \in \Lambda^{0,1}(M, V)$ if and only if $\omega(J X)=-i \omega(X)$. Assume now that $J$ is, in fact, a complex structure. Let $d$ be the usual exterior derivative operator on $T_{R}(M)$ extended to $T(M)$, then for $\omega \in \Lambda^{p, q}(M, V) d \omega$ may be written uniquely as $\partial \omega+\bar{\partial} \omega$ where $\partial \omega \in \Lambda^{p+1, q}(M, V), \bar{\partial} \omega \in \Lambda^{p, q+1}(M, V)$. In particular, if $h: M \rightarrow V$ is a differentiable map then

$$
-2 i \bar{\partial} h(X)=d h\left(J_{M} X\right)-i(d h)(X) \text { for all } X \in T(M) .
$$

Since $\bar{\partial}^{2}=0$ for a complex manifold, we have the Dolbeault cohomology groups $\mathscr{D}_{p, q}(M, V)=\operatorname{Ker} \bar{\partial}_{p, q} / \operatorname{Im} \bar{\partial}_{p, q-1}$ for all $p$ and $q$.

Let $M$ be a complex manifold, $G$ be a complex Lie group and $P$ an almost complex manifold. We say that $\xi=\{P, G, M ; \pi: P \rightarrow M\}$ is an almost holomorphic principal fibre bundle if there is an almost holomorphic action $\Phi: P \times G \rightarrow P \times P$ given by $\Phi(p, g)=\left(p, p^{g}\right)$ which is (1) an almost holomorphic diffeomorphism of $P \times G$ onto $\Phi(P \times G)$; (2) $\Phi(P \times G)$ is a closed subspace of $P \times P$ and (3) $\pi: P \rightarrow M$ induces a biholomorphic homeomorphism of $P / G$ onto $M$. We shall specify an almost holomorphic principal fibre bundle by writing $\xi: G \rightarrow P \stackrel{\pi}{\rightarrow} M$. If $P$ is a complex manifold then this is the usual definition of a holomorphic principal fibre bundle as in [13]. The equivalence of the two definitions is E. Cartan's Theorem [11, Exposé 6]. For fixed $g \in G$ the map $P \rightarrow P$ given by $p \leadsto p^{g}$ will be denoted $R_{g}$.

We say that the almost holomorphic principal fibre bundle $\xi: G \rightarrow P \stackrel{\pi}{\rightarrow} M$ is trivial as a real bundle (or is a real product) if there is a $C^{\infty}$ section (i.e., a $C^{\infty}$ map $s: M \rightarrow P$ such that $\pi \circ s$ is the identity on $M$ ). Let $\xi: G \rightarrow \widetilde{P} \stackrel{\tilde{\pi}}{\rightarrow} M$ be another almost holomorphic principal fibre bundle, then $\xi$ and $\xi$ are (holomorphically) equivalent if there is an almost holomorphic diffeomorphism $\psi: \widetilde{P} \rightarrow P$ such that

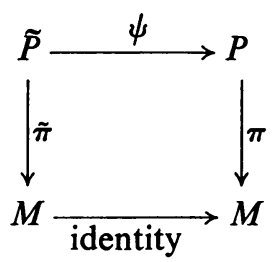

commutes and $\psi\left(\tilde{p}^{g}\right)=\psi(\tilde{p})^{g}$ for all $\tilde{p} \in \tilde{P}, g \in G$. 
Let $G \rightarrow P \stackrel{\pi}{\rightarrow} M$ be an almost holomorphic principal fibre bundle. As before, let $\operatorname{ker} \pi$ denote the sub-bundle of $T(P)$ given by $\operatorname{ker} \pi=\left\{(p, X) \mid X \in T_{p}(P), p \in P\right.$ and $\dot{\pi}(X)=0$ \}.

THEOREM 1.1.1. Let $\hat{G}$ be the Lie algebra of $G$, exp: $\hat{G} \rightarrow G$ be the usual exponential map, then there is a canonical embedding of $\hat{G}$ onto $(\operatorname{ker} \pi)_{p}$ for each $p \in P$ given by

$$
\begin{aligned}
\theta_{p}: \hat{G} & \rightarrow(\operatorname{Ker} \pi)_{p} \subset T_{p}(P) \\
A & \leadsto \theta(A), \\
\theta(A) f & =\lim _{t \rightarrow 0} \frac{f\left(p^{\exp t A}\right)-f(p)}{t} \text { for any } f: P \rightarrow C .
\end{aligned}
$$

Furthermore $\theta_{p}$ is of type $(1,0)$ (that is, $\theta_{p}(J X)=i \theta_{p}(X)$ for each $\left.X \in T_{p}(P)\right)$ and

$$
\left(R_{g}^{*} \theta\right)_{p}(A)=\theta_{p^{o}}\left(\operatorname{ad~} g^{-1}(A)\right) \text { for all } p \in P, g \in G, A \in \hat{G}
$$

where ad: $G \rightarrow$ End $(\hat{G})$ is the usual adjoint representation $\left(\operatorname{ad} g=L_{g} \circ R_{g}-1\right)$.

2. Coherent almost complex structures on fibre bundles. Let $\xi_{0}: G \rightarrow P_{J_{0}} \stackrel{\pi}{\rightarrow} M$ be an almost holomorphic principal fibre bundle, where the almost complex structure on $P$ is $J_{0}$. The almost complex structure $J$ on $P$ is said to be coherent with $\xi_{0}$ if $\xi: G \rightarrow P_{J} \stackrel{\pi}{\rightarrow} M$ is also an almost holomorphic principal fibre bundle, i.e. the action of $G$ on $P$ is holomorphic with respect to both $J_{0}$ and $J$, and the projection $\pi$ is holomorphic with respect to both $J_{0}$ and $J$.

THEOREM 1.2.1. Let $\xi_{0}: G \rightarrow P_{J_{0}} \stackrel{\pi}{\rightarrow} M$ be an almost holomorphic principal fibre bundle, then there is a 1-1 correspondence between almost complex structures on $P$ coherent with $\xi_{0}$ and $\hat{G}$-valued 1 -forms, $\omega$, on $P$ such that

(1) $\omega(\operatorname{Ker} \pi)=0$,

(2) $\left(R_{g}^{*} \omega\right)(X)=\left(\operatorname{ad} g^{-1}\right) \omega(X)$ for all $g \in G$ and $X \in T(P)$,

(3) $\omega$ is of type $(0,1)$ with respect to $J_{0}$.

Proof. Let $J$ be a coherent almost complex structure. If $X \in T_{p}(P), p \in P$, then

$$
\dot{\pi}\left(\left(J-J_{0}\right)(X)\right)=\dot{\pi}(J X)-\dot{\pi}\left(J_{0} X\right)=J_{M}(\dot{\pi} X)-J_{M}(\dot{\pi} X)=0
$$

where $J_{M}$ is the almost complex structure on $M$. We then see that $\left(J-J_{0}\right)(X)$ $\in \operatorname{Ker} \pi$ for each $X_{p} \in T(P)$, hence, by Theorem 1.1.1, there is a unique $A \in \hat{G}$ such that $\left(J-J_{0}\right)_{p}(X)=\theta_{p}(A)$. Let $\omega_{J} \in \Lambda^{1}(P, \hat{G})$ be defined by $\left(\omega_{J}\right)_{p}(X)=A$ or, more succinctly,

$$
\left(J-J_{0}\right)(X)=\theta\left(\omega_{J}(X)\right) \text { for all } X \in T(P) .
$$

We shall now show that the assignment $J \rightarrow \omega_{J}$ is a 1-1 correspondence satisfying the conditions of the theorem.

Firstly, if $X=\theta(A)$ then $\left(J-J_{0}\right)(\theta(A))=\theta(i A)-\theta(i A)=0$ so $\omega(X)=0$ for $X \in \operatorname{Ker} \pi$. 
Secondly, if $\omega_{p}(X)=A$ then

$$
J\left(R_{g} X\right)-J_{0}\left(R_{g} X\right)=R_{g}\left(\left(J-J_{0}\right) X\right)=R_{g} \theta_{p}(A)=\theta_{p^{g}}\left(\left(\operatorname{ad~} g^{-1}\right) A\right)
$$

where the last equality follows from equation (1.1.3).

Thirdly, we show that $\omega\left(J_{0} X\right)=-i \omega(X)$. By definition,

$$
\begin{gathered}
\omega_{J}(X)=A \Leftrightarrow J(X)-J_{0}(X)=\theta(A), \\
\omega_{J}\left(J_{0} X\right)=B \Leftrightarrow J\left(J_{0} X\right)-J_{0}\left(J_{0} X\right)=\theta(B) .
\end{gathered}
$$

Applying $J$ to equation (1.2.2) we obtain $-X-J J_{0} X=J \theta(A)=\theta(i A)$ so

$$
J\left(J_{0} X\right)=-\theta(i A)-X .
$$

Substituting (1.2.4) into (1.2.3) yields $(-\theta(i A)-X)+X=\theta(B)$. Hence $B=-i A$ and so $\omega\left(J_{0} X\right)=-i \omega(X)$.

Conversely: Given any $\omega \in \Lambda^{0,1}(P, \hat{G})$ satisfying (1) and (2) of the proposition, let $J^{\omega}$ be defined at $p \in P$ by

$$
\left(J^{\omega}\right)_{p}(X)=\left(J_{0}\right)_{p}(X)-\theta_{p}\left(\omega_{p}(X)\right) \text { for all } X \in T(P) .
$$

$J^{\omega}$ is clearly linear.

$$
\begin{aligned}
\left(J^{\omega}\right)^{2}(X) & =J^{\omega}\left(J_{0}(X)-\theta(\omega(X))\right) \\
& =J_{0}\left(J_{0}(X)-\theta(\omega(X))\right)-\theta\left(\omega\left(J_{0}(X)-\theta(\omega(X))\right)\right) \\
& =-X-J_{0} \theta(\omega(X))-\theta\left(\omega\left(J_{0} X\right)\right)+\theta(\omega(\theta(\omega(X)))) .
\end{aligned}
$$

But $\omega(\theta(\omega(X)))=0$ since $\theta(\omega(X))$ is vertical; hence the last term is zero so

$$
J^{2}(X)=-X-\theta(i \omega(X))+\theta(i \omega(X))=-X
$$

where the first equality follows because $\omega$ is of type $(0,1)$ with respect to $J_{0}$.

To show that $J^{\omega}$ is coherent with $\xi_{0}$ we need show that (a) $J^{\omega} \dot{R}_{g}=\dot{R}_{g} J^{\omega}$ for all $g \in G$ and (b) $\pi$ is holomorphic with respect to $J^{\omega}$.

Let $X \in T_{p}(P), p \in P$, then

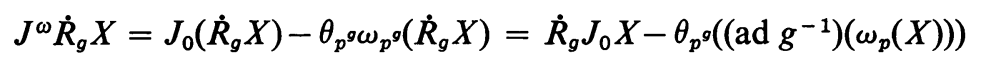

but

$$
=\dot{R}_{g} J_{0} X-\dot{R}_{g} \theta_{p}\left(\omega_{p}(X)\right)=\dot{R}_{g} J^{\omega}(X)
$$

hence (a) follows.

For $X \in T_{p}(P), \dot{\pi}\left(J^{\omega} X\right)=\dot{\pi}\left(J_{0} X-\theta_{p}\left(\omega_{p}(X)\right)\right)=\dot{\pi}\left(J_{0} X\right)-\dot{\pi}\left(\theta_{p}\left(\omega_{p}(X)\right)\right)$.

However the last term is zero because $\theta_{p}\left(\omega_{p}(X)\right) \in(\operatorname{Ker} \pi)$; hence $\dot{\pi}\left(J^{\omega} X\right)$ $=\dot{\pi}\left(J_{0} X\right)=J_{M} \dot{\pi} X$ and so $\pi$ is holomorphic with respect to $J^{\omega}$.

Corollary 1.2.2. Let $G$ be abelian, then there is a 1-1 correspondence between almost complex structures coherent with $\xi: G \rightarrow P_{J_{0}} \stackrel{\pi}{\rightarrow} M$ and elements of $\Lambda^{0,1}(M, \hat{G})$. 
Proof. By Theorem 1.2.1 we need only show that there is a 1-1 correspondence between $\hat{G}$-valued $(0,1)$-forms on $P$ such that $(1) \omega(\operatorname{Ker} \pi)=0$ and $(2)\left(R_{g}^{*} \omega\right)(X)$ $=\operatorname{ad} g^{-1}(\omega(X))=\omega(X)$ and $\Lambda^{0,1}(M, \hat{G})$. This correspondence is given as follows: Let $\eta \in \Lambda^{0,1}(M, G)$ be defined by $\eta_{m}(W)=\omega_{p}(\tilde{W})$ where $p$ is a point in $\pi^{-1}(m)$ and $\dot{\pi}(\tilde{W})=W$. Because of condition (1) above, if $\dot{\pi} \tilde{W}^{\prime}=W$ then $\omega_{p}(\tilde{W})=\omega_{p}\left(\tilde{W}^{\prime}\right)$. The definition of $\eta$ is independent of the choice of $p \in \pi^{-1}(m)$ because $\dot{\pi}_{p^{g}}\left(\dot{R}_{g} \tilde{W}\right)=\dot{\pi}_{p} \tilde{W}$ $=W$ but

$$
\omega_{p^{g}}\left(\dot{R}_{g} \tilde{W}\right)=\left(R_{g}^{*} \omega\right)_{p}(\tilde{W})=\omega_{p}(\tilde{W})
$$

It is usual to confuse the set of almost complex structures and equivalence classes of almost complex structures. Please note that Theorem 1.2.1 and the following corollaries assert nothing about equivalence classes of almost complex structures; that is, it is easy for $\omega_{1}$ and $\omega_{2} \in \Lambda^{0,1}(P, \hat{G})$ to represent equivalent almost complex structures without $\omega_{1}=\omega_{2}$. We shall investigate the notion of equivalence in Chapter II.

We shall now see which forms $\omega$ satisfying conditions (1), (2), and (3) of Theorem 1.2.1 correspond to complex structures. Let $N_{i}$ be the torsion of the almost complex structure $J_{i}(i=0,1)$; that is,

$$
N_{i}(X, Y)=2\left\{\left[J_{i} X, J_{i} Y\right]-[X, Y]-J_{i}\left[X, J_{i} Y\right]-J_{i}\left[J_{i} X, Y\right]\right\} .
$$

The Newlander-Nirenberg Theorem [10, p. 321] asserts that $J_{i}$ is a complex structure if and only if $N_{i}=0$. We shall therefore compute $N_{1}-N_{0}$ in terms of $\omega$. Note that $N_{1}-N_{0}$ is certainly vertical.

THEOREM 1.2.3. Let $\xi_{0}: G \rightarrow P_{J_{0}} \rightarrow M$ be an almost holomorphic principal fibre bundle. Let $J_{1}$ be an almost complex structure corresponding to $\omega \in \Lambda^{0,1}(P, \hat{G})$ as in Theorem 1.2.1; then

$$
\begin{array}{r}
\frac{1}{2} \theta^{-1}\left(N_{1}(X, Y)-N_{0}(X, Y)\right)=2 d \omega(J, X, Y)+2 d \omega(X, J, Y)+[\omega X, \omega Y] \\
\text { for all } X, Y \in T(P) .
\end{array}
$$

Before proving the above theorem, we shall need some notation. $A^{*} \in T(P)$ is called a fundamental vector field if there is an $A \in \hat{G}$ such that $A_{p}^{*}=\theta_{p}(A)$ for all $p \in P$. Let $\omega_{p}^{*}(X)=\theta_{p}(\omega(X)) \in \operatorname{Ker} \pi$ for each $X \in T(P)$.

LEMMA 1.2.4. For all $X, Y \in T(P)$

(a) Let $A^{*}$ be a fundamental vector field, then $J_{0}\left[A^{*}, X\right]=\left[A^{*}, J_{0} X\right]$.

(b) $\theta^{-1}\left(\left[\omega^{*} X, J_{0} Y\right]-J_{0}\left[\omega^{*} X, Y\right]\right)=i Y(\omega(X))-\left(J_{0} Y\right)(\omega(X))$.

(c) $2 d \omega\left(J_{0} X, Y\right)+2 d \omega\left(X, J_{0} Y\right)=-\omega\left(\left[X, J_{0} Y\right]+\left[J_{0} X, Y\right]\right)+\left(J_{0} X\right)(\omega(Y))-$ $J_{0} Y(\omega(X))+i Y(\omega(X))-i X(\omega(Y))$.

(d) $2 d \omega\left(\omega^{*} X, Y\right)+2 d \omega\left(X, \omega^{*} Y\right)=-\omega\left(\left[\omega^{*} X, Y\right]+\left[X, \omega^{*} Y\right]\right)+\left(\omega^{*} X\right)(\omega Y)-$ $\left(\omega^{*} Y\right)(\omega X)$.

(e) $\theta^{-1}\left(\left[\omega^{*} X, \omega^{*} Y\right]\right)=\left(\omega^{*} X\right)(\omega Y)-\left(\omega^{*} Y\right)(\omega X)+[\omega X, \omega Y]$. 
Proof. (a) Let $\phi_{t}(p)=p^{\exp t A}$ for all $p \in P, A \in \hat{G}, t \in R$, then $\phi_{t}$ is the one parameter group of diffeomorphisms of $P$ generated by $A^{*}$; hence $[9$, p. 15]

$$
J_{0}\left[A^{*}, X\right]=J_{0}\left(\lim _{t \rightarrow 0} \frac{1}{t}\left(X-\dot{\phi}_{t}\right) X\right) .
$$

Since the action of $G$ on $P$ is almost holomorphic we have $J_{0} \dot{\phi}_{t}=\dot{\phi}_{t} J_{0}$; hence

$$
J_{0}\left[A^{*}, X\right]=\lim _{t \rightarrow 0} \frac{1}{t}\left(J_{0} X-\dot{\phi}_{t}\left(J_{0} X\right)\right)=\left[A^{*}, J_{0} X\right] .
$$

(b) Let $\omega^{*} X=\sum f^{t} A_{i}^{*}$ where $f^{i}$ are smooth functions on $P$, then (using part (a))

$$
\begin{aligned}
\theta^{-1}\left(\left[\omega^{*} X, J_{0} Y\right]-J_{0}\left[\omega^{*} X, Y\right]\right)= & \sum \theta^{-1}\left\{\left[f^{i} A_{i}^{*}, J_{0} Y\right]-J_{0}\left[f^{i} A_{i}^{*}, Y\right]\right\} \\
= & \sum \theta^{-1}\left\{f^{i}\left[A_{i}^{*}, J_{0} Y\right]-\left(J_{0} Y\right) f^{i} A_{i}^{*}+f^{i}\left(J_{0} Y\right) A_{i}^{*}\right. \\
& \left.\quad-J_{0}\left(f^{4}\left[A_{i}^{*}, Y\right]-Y f^{i} A_{i}^{*}+f^{i} Y A_{i}^{*}\right)\right\} \\
= & -\left(J_{0} Y\right) \omega(X)+i Y \omega(X) .
\end{aligned}
$$

(c) $2 d \omega\left(J_{0} X, Y\right)+2 d \omega\left(X, J_{0} Y\right)$

$$
\begin{aligned}
& =J_{0} X \omega(Y)-Y \omega\left(J_{0} X\right)-\omega\left(\left[J_{0} X, Y\right]\right)+X \omega\left(J_{0} Y\right)-J_{0} Y \omega(X)-\omega\left(\left[X, J_{0} Y\right]\right) \\
& =J_{0} X \omega(Y)+i Y \omega(X)-i X \omega(Y)-J_{0} Y \omega(X)-\omega\left(\left[J_{0} X, Y\right]+\left[X, J_{0} Y\right]\right)
\end{aligned}
$$

where the last equality follows because $\omega \in \Lambda^{0.1}(P, \hat{G})$.

(d) $2 d \omega\left(\omega^{*} X, Y\right)+2 d \omega\left(X, \omega^{*} Y\right)$

$$
\begin{aligned}
= & \left(\omega^{*} X\right) \omega(Y)-Y \omega\left(\omega^{*} X\right)-\omega\left(\left[\omega^{*} X, Y\right]\right) \\
& +X \omega\left(\omega^{*} Y\right)-\left(\omega^{*} Y\right) \omega(X)-\omega\left(\left[X, \omega^{*} Y\right]\right) \\
= & -\omega\left(\left[\omega^{*} X, Y\right]+\left[X, \omega^{*} Y\right]\right)+\left(\omega^{*} X\right)(\omega Y)-\left(\omega^{*} Y\right)(\omega X)
\end{aligned}
$$

since $\omega(\operatorname{Ker} \pi)=0$.

(e) Let $\omega^{*} X=\sum_{i} f^{i} A_{i}^{*}$ and $\omega^{*} Y=\sum_{j} g^{j} A_{j}^{*}$ then $\omega X=\sum_{i} f^{i} A_{i}$ and $\omega Y=\sum_{j} g^{j} A_{j}$; hence

$$
\begin{aligned}
\left(\omega^{*} X\right)(\omega Y)-\left(\omega^{*} Y\right)(\omega X) & +[\omega X, \omega Y] \\
& =\sum_{i, j}\left\{\left(f^{i} A_{i}^{*}\right)\left(g^{j} A_{j}\right)-\left(g^{j} A_{j}^{*}\right)\left(f^{i} A_{i}\right)-\left[f^{i} A_{i}, g^{j} A_{j}\right]\right\} ;
\end{aligned}
$$

hence, since the last bracket is in $\hat{G}$,

$$
=\sum_{i, j}\left\{f^{i}\left(A_{i}^{*} g^{j}\right) A_{j}-g^{j}\left(A_{j}^{*} f^{i}\right) A_{i}-f^{i} g^{j}\left[A_{i}, A_{j}\right]\right\} .
$$

Thus

$$
\begin{aligned}
& \theta\left(\left(\omega^{*} X\right)(\omega Y)-\left(\omega^{*} Y\right)(\omega X)+[\omega X, \omega Y]\right) \\
& \quad=\sum_{i, j}\left\{f^{t}\left(A_{i}^{*} g^{j}\right) A_{j}^{*}-g^{j} A_{j}^{*} f^{i} A_{i}^{*}-f^{t} g^{j}\left[A_{i}^{*}, A_{j}^{*}\right]\right\}=\left[\omega^{*}(X), \omega^{*}(Y)\right] .
\end{aligned}
$$


We shall now prove Theorem 1.2.3. Because $\left(J_{1}-J_{0}\right)(X)=\theta(\omega(X))=\omega^{*}(X)$ we have

$$
\begin{aligned}
& \frac{1}{2} N_{1}(X, Y) \\
& =\left[J_{0} X+\omega^{*} X, J_{0} Y+\omega^{*} Y\right]-[X, Y]-J_{1}\left[X, J_{0} Y+\omega^{*} Y\right]-J_{1}\left[J_{0} X+\omega^{*} X, Y\right] \\
& =\left[J_{0} X, J_{0} Y\right]+\left[\omega^{*} X, \omega^{*} Y\right]+\left[J_{0} X, \omega^{*} Y\right]+\left[\omega^{*} X, J_{0} Y\right] \\
& -[X, Y]-J_{0}\left[X, J_{0} Y\right]-J_{0}\left[X, \omega^{*} Y\right]-\omega^{*}\left(\left[X, J_{0} Y\right]\right) \\
& -\omega^{*}\left(\left[X, \omega^{*} Y\right]\right)-J_{0}\left[J_{0} X, Y\right]-J_{0}\left[\omega^{*} X, Y\right]-\omega^{*}\left[J_{0} X, Y\right]-\omega^{*}\left[\omega^{*} X, Y\right] \text {. } \\
& \frac{1}{2} N_{1}(X, Y)=\frac{1}{2} N_{0}(X, Y)+\left\{\left[\omega^{*} X, \omega^{*} Y\right]-\omega^{*}\left[X, \omega^{*} Y\right]-\omega^{*}\left[\omega^{*} X, Y\right]\right\} \\
& +\left\{\left[\omega^{*} X, J_{0} Y\right]-J_{0}\left[\omega^{*} X, Y\right]\right\}+\left\{\left[J_{0} X, \omega^{*} Y\right]-J_{0}\left[X, \omega^{*} Y\right]\right\} \\
& -\left\{\omega^{*}\left(\left[X, J_{0} Y\right]\right)+\omega^{*}\left(\left[J_{0} X, Y\right]\right)\right\} \text {. }
\end{aligned}
$$

We shall now reduce the right-hand side of equation (1.2.5) to the above form. $2 d \omega\left(J_{1} X, Y\right)+2 d \omega\left(X, J_{1} Y\right)+[\omega X, \omega Y]$

$$
=2 d \omega\left(\omega^{*} X, Y\right)+2 d \omega\left(X, \omega^{*} Y\right)+2 d \omega\left(J_{0} X, Y\right)+2 d \omega\left(X, J_{0} Y\right)+[\omega X, \omega Y] \text {. }
$$

Applying Lemma 1.2.4(c), (d):

$$
\begin{aligned}
= & -\omega\left\{\left[\omega^{*} X, Y\right]+\left[X, \omega^{*} Y\right]\right\}+\left(\omega^{*} X\right)(\omega Y)-\left(\omega^{*} Y\right)(\omega X) \\
& -\omega\left\{\left[X, J_{0} Y\right]+\left[J_{0} X, Y\right]\right\}+\left(J_{0} X\right) \omega(Y)-\left(J_{0} Y\right) \omega(X) \\
& +i Y(\omega X)-i X \omega Y+[\omega X, \omega Y] .
\end{aligned}
$$

Applying Lemma 1.2.4(b), (e) yields:

$$
\begin{aligned}
= & -\omega\left(\left[\omega^{*} X, Y\right]+\left[X, \omega^{*} Y\right]\right)+\theta^{-1}\left(\left[\omega^{*} X, \omega^{*} Y\right]\right) \\
& -\omega\left(\left[X, J_{0} Y\right]+\left[J_{0} X, Y\right]\right)+\theta^{-1}\left(\left[J_{0} X, \omega^{*} Y\right]-J_{0}\left[X, \omega^{*} Y\right]\right) \\
& +\theta^{-1}\left(\left[\omega^{*} X, J_{0} Y\right]-J_{0}\left[\omega^{*} X, Y\right]\right) .
\end{aligned}
$$

Since $\omega=\theta^{-1} \omega^{*}$ we have

$2 d \omega\left(J_{1} X, Y\right)+2 d \omega\left(X, J_{1} Y\right)+[\omega X, \omega Y]$

$$
\begin{aligned}
=\theta^{-1}\left\{\left[\omega^{*} X, \omega^{*} Y\right]-\omega^{*}\left[X, \omega^{*} Y\right]-\omega^{*}\left[\omega^{*} X, Y\right]+\left[\omega^{*} X, J_{0} Y\right]\right. \\
\left.-J_{0}\left[\omega^{*} X, Y\right]+\left[J_{0} X, \omega^{*} Y\right]-J_{0}\left[X, \omega^{*} Y\right]-\omega^{*}\left[X, J_{0} Y\right]-\omega^{*}\left[J_{0} X, Y\right]\right\}
\end{aligned}
$$

which is exactly $\theta^{-1}$ applied to the right-hand side of equation (1.2.6).

THEOREM 1.2.5. Let $\xi_{0}: G \rightarrow P_{J_{0}} \rightarrow M$ be a holomorphic principal fibre bundle. Let $J_{1}$ be an almost complex structure coherent with $J_{0}$. If $J_{1}$ corresponds to $\omega \in \Lambda^{0,1}(P, \hat{G})$ then $J_{1}$ is a complex structure if and only if $\bar{\partial} \omega+(d \omega)_{2,0}=-(i / 4)[\omega, \omega]$. In particular, if $J_{1}$ is complex then $\bar{\partial}_{J_{1}} \omega=-(i / 4)[\omega, \omega]$.

Proof. Assume that $J_{1}$ is a complex structure, then equation (1.2.5) implies that $2 d \omega\left(J_{1} X, Y\right)+2 d \omega\left(X, J_{1} Y\right)=-[\omega(X), \omega(Y)]$. Let

$$
-\psi(X, Y)=2 d \omega\left(J_{1} X, Y\right)+2 d \omega\left(X, J_{1} Y\right) ;
$$


then it suffices to show $\psi=4 i \bar{\partial} \omega$. Since $J_{1}$ is complex, $d \omega \in \Lambda_{J_{1}}^{0,2}(P, \hat{G}) \oplus \Lambda_{J_{1}}^{1,1}(P, \hat{G})$. If $X, Y \in T_{J_{1}}^{1,0}(P)$ then $\psi(X, Y)=4 i d \omega(X, Y)=0$. If $X \in T_{J_{1}}^{1,0}(P)$ and $Y \in T_{J_{1}}^{0,1}(P)$ then $\psi(X, Y)=2 i d \omega(X, Y)-2 i d \omega(X, Y)=0$, therefore $\psi \in \Lambda^{(0,2)}(P, \hat{G})$. Now $d \omega-\psi / 4 i$ has no $(0,2)$ component because if $X, Y \in T_{J_{1}}^{0,1}(P)$ then

$$
\begin{aligned}
d \omega(X, Y)-\psi(X, Y) & =d \omega(X, Y)-(2 i d \omega(X, Y)+2 i d \omega(X, Y)) / 4 i \\
& =d \omega(X, Y)-d \omega(X, Y)=0 .
\end{aligned}
$$

Since $d \omega=(d \omega-\psi / 4 i)+\psi / 4 i, \psi / 4 i$ is of type $(0,2)$ and $d \omega-\psi / 4 i$ has no component of type $(0,2)$ we conclude that $\psi / 4 i$ is the $(0,2)$ component of $d \omega$, i.e. $\bar{\partial} \omega=\psi / 4 i$ because $\omega$ is of type $(0,1)$ with respect to $J_{1}$ as well as $J_{0}$.

We shall now prove that if $(i / 4)[\omega, \omega]$ is the sum of the $(0,2)$ and $(2,0)$ components of $d \omega$ then $J_{1}$ is in fact a complex structure. By a procedure similar to the previous one we see that $2 d \omega\left(J_{1} X, Y\right)+2 d \omega\left(X, J_{1} Y\right)$ is $4 i$ times the sum of the $(0,2)$ and $(2,0)$ components of $d \omega$ hence $2 d \omega\left(J_{1} X, Y\right)+2 d \omega\left(X, J_{1} Y\right)+[\omega X, \omega Y]$ $=0$ and so $N_{1}=0$ by Theorem 1.2.2.

We shall now examine the real trivial case. If $\xi: G \rightarrow P \stackrel{\pi}{\rightarrow} M$ is an almost holomorphic principal fibre bundle then we say that $\xi$ is real product if $\xi$ admits a $C^{\infty}$ section; that is, a smooth map $s: M \rightarrow P$ such that $\pi \circ s$ is the identity map on $M$. Clearly if $\xi$ is real product then $P$ is diffeomorphic to $M \times G$. Let $J$ be the complex structure on $M \times G$ given by $J_{z, \lambda}(W, Z)=\left(J_{M} W, J_{G} Z\right)$ for all $m \in M, \lambda \in G$, $W \in T_{m}(M)$ and $Z \in T_{\lambda}(G)$; then $J$ is called the product complex structure on $M \times G$. Clearly, a bundle $\xi$ is a real product if and only if it is coherent with the product structure on $M \times G$. We now determine all real trivial almost holomorphic principal fibre bundles.

THEOREM 1.2.6. There is a one-to-one correspondence between $\Lambda^{0,1}(M, \hat{G})$ and almost complex structures on $M \times G$ coherent with the product structure. In fact, this correspondence is given by $\eta \in \Lambda^{0,1}(M, \hat{G}), \eta \leadsto J^{\eta}$, where

$$
J_{z, \lambda}^{\eta}(W, Z)=\left(J_{M} W, J_{G} Z+\left(d R_{\lambda}\right)_{e} \eta(W)\right)
$$

for $z \in M, \lambda \in G, W \in T_{z}(M), Z \in T_{\lambda}(G)$.

Proof. The canonical embedding $\theta$ of $\hat{G}$ into $T(P)=T(M \times G)=T(M) \times T(G)$ takes the form $\theta_{z, \lambda}(A)=\left(0,\left(d R_{\lambda}\right)(A)\right), A \in \hat{G}, z \in M, \lambda \in G$. Thus let $X=(W, Z)$ $\in T_{z, \lambda}(M \times G)$; then equation (1.2.1) reduces to (with $J_{0}=$ product structure)

$$
\omega_{J}(X)=A \Leftrightarrow J(X)-\left(J_{M} W, J_{G} Z\right)=\left(0,\left(d R_{\lambda}\right)_{e}(A)\right),
$$

i.e. by Theorem 1.2.1 there is a 1-1 correspondence between those $\omega \in \Lambda^{0.1}(P, \hat{G})$ such that $\omega(\operatorname{Ker} \pi)=0$ and $R_{g}^{*} \omega=\operatorname{ad~} g^{-1} \omega$ with almost complex structures on $M \times G$ coherent with the product structure and this correspondence is given by equation (1.2.10). We need only show that there is a 1-1 correspondence between those $\omega \in \Lambda^{0,1}(P, \hat{G})$ such that $\omega(\operatorname{Ker} \pi)=0$ and $R_{g}^{*} \omega=\operatorname{ad} g^{-1} \omega$ with $\Lambda^{0,1}(M, \hat{G})$. This is provided by the following lemma. 
Lemma 1.2.7. If $\xi: G \rightarrow P \rightarrow M$ is a real trivial holomorphic principal fibre bundle then there is a one-to-one correspondence between those elements $\omega \in \Lambda^{0,1}(P, \hat{G})$ such that (a) $\omega(\operatorname{Ker} \pi)=0$, (b) $R_{g}^{*} \omega=\left(\operatorname{ad} g^{-1}\right) \omega$ and the set of all $\eta \in \Lambda^{0,1}(M, \hat{G})$.

Proof. Let $s: M \rightarrow P$ be a $C^{\infty}$ section on $\xi$. We may assume $P$ is diffeomorphic to $M \times G$, and $s: M \rightarrow M \times G$ is given by $s(m)=(m, e)$ where $e$ is the identity of $G$. Thus $s^{*}: \Lambda^{0,1}(P, \hat{G}) \rightarrow \Lambda^{0,1}(M, \hat{G})$. We shall prove that this map is a $1-1$ correspondence as in the lemma.

We first show that $s^{*}\left(\Lambda^{0,1}(P, \hat{G})\right) \subset \Lambda^{0,1}(M, \hat{G})$. Let $\omega \in \Lambda^{0,1}(P, \hat{G})$; then

$$
\left(s^{*} \omega\right)\left(J_{M} A\right)=\omega\left(d s\left(J_{M} A\right)\right)=\omega\left(\left(J_{M} A, 0\right)\right)=-i \omega(A, 0)=-i\left(s^{*} \omega\right)(A),
$$

so $s^{*} \omega \in \Lambda^{0,1}(M, \hat{G})$.

Suppose $s^{*} \omega=0$, then $\omega_{z, e}((A, 0))=0$ for all $A \in T_{z}(M)$. However, by assumption $\omega_{z, e}((0, B))=0$; hence $\omega_{z, e}(A, B)=\omega_{z, e}(A, 0)+\omega_{z, e}(0, B)=0$. However,

$$
\omega_{z, \lambda}(A, B)=\left(\operatorname{ad} \lambda^{-1}\right) \omega_{z, e}(A, B)=0 .
$$

We shall now show that $s^{*}$ is onto. Let $\eta \in \Lambda^{0,1}(M, \hat{G})$. Let $\omega_{z, \lambda}(A, B)$ $=\left(\operatorname{ad} \lambda^{-1}\right) \eta_{z}(A) ;$ then $\omega$ satisfies all of the necessary conditions.

\section{Chapter II: Complex Structures on Real Product Bundles}

1. Statement of the problem and solution in the abelian case. The aim of this chapter is to obtain information on the set of (holomorphic) equivalence classes of principal fibre bundles with structure group $G$ and base $M$ which are trivial as smooth bundles, i.e. equivalence classes of real product bundles.

We shall call this set Pic $(M, G)$. If $M$ is a Riemann surface and $G=C^{*}$ then Pic $(M, G)$ is called the Picard variety of $M$ [7]. We shall now give the sheaftheoretic interpretation of Pic $(M, G)$. Let $H^{1}\left(M, G_{h}\right)$ (resp. $H^{1}\left(M, G_{\infty}\right)$ ) denote the first cohomology set of $M$ with coefficients in the sheaf of germs of holomorphic (resp. $C^{\infty}$ ) maps from $M$ to $G$. If $\alpha: \boldsymbol{G}_{h} \rightarrow \boldsymbol{G}_{\infty}$ is the map obtained by viewing a holomorphic map as a $C^{\infty}$ map, then $\alpha$ induces a map, also called $\alpha: H^{1}\left(M, G_{h}\right)$ $\rightarrow H^{1}\left(M, G_{\infty}\right)$. It is well known [4] that the set of holomorphic (resp. $C^{\infty}$ ) equivalence classes of holomorphic (resp. $C^{\infty}$ ) principal fibre bundles over $M$ with structure group $G$ is equal to $H^{1}\left(M, G_{h}\right)$ (resp. $H^{1}\left(M, G_{\infty}\right)$ ) where the trivial holomorphic (resp. $C^{\infty}$ ) bundle is denoted by $O_{h}$ (resp. $O_{\infty}$ ); that is, $O_{h}: G \rightarrow(M \times G) \rightarrow$ Product structure $M$. The set of equivalence classes of real trivial holomorphic principal fibre bundles is therefore $\operatorname{ker} \alpha=\alpha^{-1}\left(O_{\infty}\right)$.

We will first explain why Pic $(M, G)$ need not be zero.

Topologically. $\xi: G \rightarrow(M \times G)_{J} \rightarrow M$ is the general form of a real product bundle where the action of $G$ on $(M \times G)_{J}$ is, of course, the second coordinate action (i.e. $(z, g)^{\lambda}=(z, g \lambda)$ for all $\left.z \in M, g, \lambda \in G\right)$. In order for $\xi$ to be holomorphically trivial, the complex structure $J$ must be equivalent to the product of the complex structures of $M$ and $G$. We will see below that $M \times G$ can be given complex structures which are not equivalent to the product structure. 
Analytically. We now view a bundle $\xi$ in terms of its transition functions [13]. $\xi$ is trivial as a $C^{\infty}$-bundle if and only if there is an open cover $\left\{U_{\alpha}\right\}$ of $M$ such that the transition functions $\left\{t_{\alpha \beta}: U_{\alpha} \cap U_{\beta} \rightarrow G\right\}$ of $\xi$ with respect to $\left\{U_{\alpha}\right\}$ take the form

$$
t_{\alpha \beta}(z)=s_{\alpha}(z) s_{\beta}(z)^{-1}, \quad z \in U_{\alpha} \cap U_{\beta},
$$

where $s_{\alpha}: U_{\alpha} \rightarrow G$ and $s_{\beta}: U_{\beta} \rightarrow G$ are smooth functions. Because $\xi$ is a holomorphic bundle the $t_{\alpha \beta}$ are holomorphic functions but, of course, the $s_{\alpha}$ need not be holomorphic. In fact, there are holomorphic functions satisfying (2.1.1) exactly when $\xi$ is trivial as a holomorphic bundle. In the case when $G=C^{*}$, the problem of finding holomorphic functions $s_{\alpha}$ satisfying (2.1.1) (given the holomorphic functions $\left.t_{\alpha \beta}\right)$ is called the Cousin problem of several complex variables, and the phrasing of this problem in fibre bundle terminology was first published by H. Cartan [3].

Recall that there is an isomorphism (due to Dolbeault-Serre, [4])

$$
\text { dolbeault: } \mathscr{D}_{p, q}(M, V) \stackrel{\sim}{\longrightarrow} H^{q}\left(M, \Omega^{p}(V)\right)
$$

where $V$ is any vector bundle over $M$ and $\Omega^{p}(V)$ is the sheaf of germs of holomorphic sections of $\Lambda^{p, 0}(M, V)$. For any details about the arguments in sheaf-theoretic cohomology which follow see [4].

When $G$ is abelian, we can obtain the following information: (If $M$ is a compact Riemann surface and $G=C^{*}=\{z \in C \mid z \neq 0\}$ then this result is in [7].)

THEOREM 2.1.1. Let $M$ be a complex manifold, $G$ an abelian complex Lie group; then the following is an exact sequence of abelian groups

$$
\begin{aligned}
\stackrel{i}{\longrightarrow} \mathscr{D}_{0,1}(M, \hat{G}) \stackrel{\beta}{\longrightarrow} H^{1}\left(M, G_{h}\right) \stackrel{\alpha}{\longrightarrow} H^{1}\left(M, G_{\infty}\right) \\
\stackrel{i \circ \delta_{\infty}}{\longrightarrow} H^{2}\left(M, \pi_{1}(G)\right) \longrightarrow \mathscr{D}_{0,2}(M, \hat{G}) \longrightarrow
\end{aligned}
$$

where $\beta=\exp \circ$ dolbeault.

Proof. Because exp: $\hat{G} \rightarrow G$ is a covering map (recall all Lie groups are assumed to be connected), we see that the sequence of abelian groups $0 \longrightarrow \pi_{1}(G) \longrightarrow \hat{G}$ $\stackrel{\text { exp }}{\longrightarrow} \longrightarrow 0$ is exact; hence so are the sequences in cohomology:

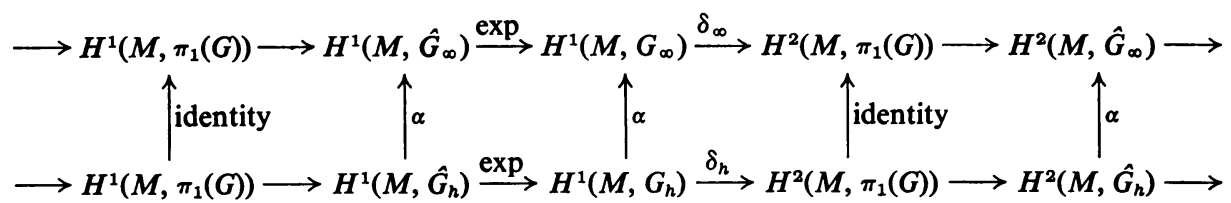

However $\hat{G}$ is isomorphic as a Lie group to $\boldsymbol{C}^{n}$, hence $\hat{\boldsymbol{G}}_{\infty}$ is a fine sheaf and so $H^{k}\left(M, \hat{G}_{\infty}\right)=0$ for all $k \geqq 1$, hence $\delta_{\infty}$ is an isomorphism. The Dolbeault isomorphism and some diagram chasing completes the proof.

COROLlary 2.1.2. Let $G$ be abelian; then $\operatorname{Pic}(M, G)$ has the natural structure of the product of a torus with $C^{r}$. 
Proof. The proof is by diagram chasing.

$$
\operatorname{Pic}(M, G)=\operatorname{ker} \alpha=\text { image } \beta=\mathscr{D}_{0,1}(M, \hat{G}) / i\left(H^{1}\left(M, \pi_{1}(G)\right)\right) \text {. }
$$

Since $\pi_{1}(G)$ is free, $i\left(H^{1}\left(M, \pi_{1}(G)\right)\right)$ is a lattice in the vector space $\mathscr{D}_{0,1}(M, G)$, i.e. Pic $(M, G)$ is the quotient of $C^{n}$ ( $n$ might be infinite) by a discrete subgroup, hence has the structure of a torus crossed with $C^{r}$.

CoRollary 2.1.3. If $\pi_{1}(M)$ is a torsion group and $G$ is abelian then $\operatorname{Pic}(M, G)$ is isomorphic to $\mathscr{D}_{0,1}(M, \hat{G})$.

Proof. The universal coefficient theorem for cohomology [12] states that

$$
0 \rightarrow \operatorname{Ext}\left(H_{0}(M), \pi_{1}(G)\right) \rightarrow H^{1}\left(M, \pi_{1}(G)\right) \rightarrow \operatorname{Hom}\left(H_{1}(M), \pi_{1}(G)\right) \rightarrow 0
$$

is exact. However $H_{0}(M)$ is a free group, so $\operatorname{Ext}\left(H_{0}(M), \pi_{1}(G)\right)=0$ and $H_{1}(M)$ is torsion (by the Hurewicz isomorphism); hence we conclude $H^{1}\left(M, \pi_{1}(G)\right)=0$ because there are no nonzero homomorphisms from a torsion group to a free group.

Note that if $\pi_{1}(M)$ is a torsion group and $G$ is abelian then Pic $(M, G)$ has a natural structure of a (infinite-dimensional) vector space. We shall see that this will contrast violently with the simplest nonabelian case in which $\operatorname{Pic}(M, G)$ is either zero or is not even Hausdorff!

2. Statement and proof of the fundamental theorem. Before starting the main theorem (Theorem 2.2.4), we shall require some definitions. As always, $M$ will be a connected complex manifold and $G$ a connected complex Lie group with Lie algebra $\hat{G}$. We say that $G$ has the exponential lift property with respect to $M$ if for any $\left(C^{\infty}\right)$ map $s: M \rightarrow G$ there is a $\left(C^{\infty}\right)$ map $h: M \rightarrow G$ such that the following diagram commutes:

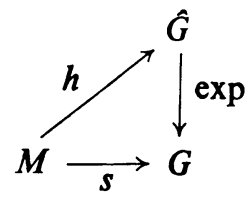

We say that a solvable Lie group is of type (E) if the exponential map is a covering map. ( $G$ is of type (E) if $G$ is nilpotent. If $G$ is solvable there is a criterion due to Dixmier; see [2] for details.)

Proposition 2.2.2. If $\pi_{1}(M)$ is a torsion group and $G$ is of type (E), then $G$ has the exponential lift property with respect to $M$.

Proof. It is well known, [12], that given $s: M \rightarrow G$ as in (2.2.1) there is $h: M \rightarrow \hat{G}$ such that (2.2.1) commutes if and only if $s_{\#}\left(\pi_{1}(M)\right) \subset \exp _{\#}\left(\pi_{1}(\hat{G})\right)=0$ where \# denotes the map induced on the fundamental group $\pi_{1}$. Since $G$ is solvable, $\pi_{1}(G)$ is free, but the image under $s_{\#}$ of the torsion group $\pi_{1}(M)$ is torsion and hence is zero, so $s: M \rightarrow G$ always lifts. 
If $\omega, \eta \in \Lambda^{0,1}(M, \hat{G})$, then we say that $\omega$ is exponentially cohomologous (or, more briefly, expcohomologous) to $\eta$ (and write $\omega \sim \exp \eta$ ) if there is a map $h: M \rightarrow \hat{G}$ such that

$$
\begin{aligned}
& 2 i\left(\left(I-e^{-\operatorname{ad} h(z)}\right) / \operatorname{ad} h(z)\right)(\bar{\partial} h(A))=\omega(A)-e^{-\operatorname{ad} h(z)} \eta(A) \\
& \quad \text { for all } A \in T_{z}(M), z \in M .
\end{aligned}
$$

The first term is to be interpreted (for each $z \in M$ ) as the convergent series

$$
\sum_{K=0}^{\infty} \frac{(-1)^{K}(\operatorname{ad} h(z))^{K}}{(K+1) !} \in \operatorname{End}(G)
$$

all forms are evaluated at $z \in M$ and $(\operatorname{ad} h(u))(X)=[h(u), X]$ for all $X \in \hat{G}$. Note that $\sim_{\text {exp }}$ is reflexive $(h=0)$ and symmetric (let $f=-h$ ). I have not been able to show that $\sim_{\text {exp }}$ is transitive. We shall use the same symbol to denote the equivalence relation generated by the relation $\sim_{\exp }$ of equation (2.2.2). $\omega$ is exponentially exact (or expexact) if $\omega \sim_{\exp } 0 . \omega$ is exponentially closed (or expclosed) if there is an open cover $\left\{U_{\alpha}\right\}$ of $M$ such that $i_{\alpha}^{*} \omega$ is expexact $\left(i_{\alpha}: U_{\alpha} \rightarrow M\right.$ is the obvious inclusion). Let $Z \exp (M, \hat{G})$ be the set of expclosed $(0,1)$-forms. We define

$$
A \operatorname{Exp} D(M, G)=\Lambda^{0,1}(M, \hat{G}) / \sim_{\text {exp }}, \quad \operatorname{Exp} D(M, G)=Z \exp (M, \hat{G}) / \sim_{\text {exp }},
$$

then both $A \operatorname{Exp} D$ and $\operatorname{Exp} D$ are sets with a distinguished element 0 (being the equivalence class of the $(0,1)$-form, $\omega \equiv 0)$.

Proposition 2.2.3. Let $G$ be abelian; then $\operatorname{Exp} D(M, G)=\mathscr{D}_{0,1}(M, \hat{G})$.

Proof. If $G$ is abelian then $\operatorname{ad} h(z)=0$, hence equation (2.2.2) becomes $2 i \bar{\partial} h$ $=\omega-\eta$; hence $\omega \sim \exp \eta$ if and only if $\omega$ is $\bar{\partial}$-cohomologous to $\eta . Z \exp (M, \hat{G})$ is just the $\bar{\partial}$-cocycles by the Dolbeault-Grothendieck Lemma.

We shall now state the main theorem of this chapter. We shall refer to this as the fundamental theorem.

THEOREM 2.2.4. Let $M$ be a complex manifold, $G$ a complex Lie group. Let

$$
\begin{gathered}
\Xi: \operatorname{Exp} D(M, \hat{G}) \rightarrow \operatorname{Pic}(M, G) \\
{[\omega] \leadsto J^{\omega}}
\end{gathered}
$$

be defined by

$$
J_{z, \lambda}^{\omega}(A, B)=\left(J_{M} A, J_{G} B+\left(d R_{\lambda}\right) \omega(A)\right)
$$

(as in equation (1.2.9)); then

(1) $\Xi$ is onto.

(2) If $G$ has the exponential lift property with respect to $M$, then $\Xi$ is an isomorphism.

It will also be clear from the proof of this theorem that if $G$ has the exponential lift property with respect to $M$ then $\sim_{\text {exp }}$ is a true equivalence relation. 
We begin with a lemma. Let $\xi: G \rightarrow(M \times G)_{J_{1}} \rightarrow M$ and $\psi: G \rightarrow(M \times G)_{J_{2}} \rightarrow M$ be real trivial holomorphic principal fibre bundles. Let $J_{1}$ correspond to $\omega$ and $J_{2}$ correspond to $\eta$ via Theorem 1.2.6, i.e. $J_{1}=J^{\omega}$ and $J_{2}=J^{\eta}$ in the notation of equations (1.2.9) and (2.2.3).

LEMMA 2.2.5. $\xi$ is equivalent to $\psi$ if and only if there exists a smooth map $\gamma: M \rightarrow G$ such that

$$
\begin{array}{r}
J_{G} d \gamma_{z}(A)+d R_{\gamma(z)}\left(\eta_{z}(A)\right)=d \gamma_{z}\left(J_{G} A\right)+d L_{\gamma(z)}\left(\omega_{z}(A)\right) \\
\qquad \text { for all } A \in T_{z}(M) \text { and all } z \in M .
\end{array}
$$

Proof. $\xi$ is equivalent to $\psi$ if and only if $\exists \phi:(M \times G)_{J_{1}} \rightarrow(M \times G)_{J_{2}}$ which is an equivariant almost holomorphic diffeomorphism which induces the identity on $M$, i.e. the following diagram commutes:

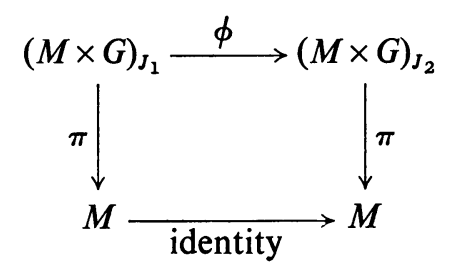

Condition (I) holds $\Leftrightarrow \phi(m, g)=(m, \beta(m, g))$ for some $\beta: M \times G \rightarrow G$. Now condition (II) says $\phi\left(m, g_{1}\right) g_{2}=\left(m, \beta\left(m, g_{1}\right) g_{2}\right)=\phi\left(m, g_{1} g_{2}\right)=\left(m, \beta\left(m, g_{1} g_{2}\right)\right)$ so $\beta\left(m, g_{1}\right) g_{2}=\beta\left(m, g_{1} g_{2}\right)$. If $\beta(m, e)=\gamma(m)$, then letting $g_{1}=e, g_{2}=g$ shows that $\beta(m, e) g=\beta(m, g)$ and so $\phi$ satisfies (I) and (II) if and only if there is a $\gamma: M \rightarrow G$ such that $\phi(m, g)=(m, \gamma(m) g)$. We shall now calculate $\dot{\phi}$ and test condition (III):

$$
\dot{\phi}_{z, \lambda}(A, B)=\left(A, \dot{\beta}_{z, \lambda}(A, B)\right), \quad A \in T_{z}(M), B \in T_{\lambda}(G),
$$

where $\beta(m, g)=\gamma(m) g$. Let ${ }^{z} \beta: G \rightarrow G$ be ${ }^{z} \beta(g)=\gamma(z) g$ and $\beta^{\lambda}: M \rightarrow M$ be $\beta^{\lambda}(m)$ $=\gamma(m) \lambda$; then ${ }^{z} \beta=L_{\gamma(z)}$ and $\beta^{\lambda}=R_{\lambda} \circ \gamma$. The Leibniz formula [9] dictates:

$$
\dot{\beta}_{z, \lambda}(A, B)=\left({ }^{z} \dot{\beta}\right)_{\lambda}(B)+\left(\dot{\beta}^{\lambda}\right)_{z}(A)
$$

or

$$
\dot{\beta}_{z, \lambda}(A, B)=\dot{L}_{\gamma(z)}(B)+\left(R_{\lambda} \circ \gamma\right)_{z}(A)
$$

Now

so

$$
\dot{\phi}_{z, \lambda}\left(J_{1}(A, B)\right)=\dot{\phi}_{z, \lambda}\left(J_{M} A, J_{G} B+\dot{R}_{\lambda} \omega(A)\right)
$$

$$
\dot{\phi}_{z, \lambda}\left(J_{1}(A, B)\right)=\left(J_{M} A, \dot{\beta}_{z, \lambda}\left(J_{M} A, J_{G} B+\dot{R}_{\lambda} \omega(A)\right)\right)
$$

and

$$
J_{2} \dot{\phi}_{z, \lambda}(A, B)=J_{2}\left(A, \dot{\beta}_{z, \lambda}(A, B)\right)=\left(J_{M} A, J_{G} \dot{\beta}_{z, \lambda}(A, B)+\dot{R}_{\gamma(z) \lambda}(\eta(A))\right)
$$


Thus $\dot{\phi} J_{1}=J_{2} \dot{\phi}$ if and only if

$$
i \dot{\beta}_{z, \lambda}(A, B)+\dot{R}_{\gamma(z) \lambda} \eta(A)=\dot{\beta}_{z, \lambda}\left(J_{M} A, J_{G} B+\dot{R}_{\lambda} \omega(A)\right) .
$$

Hence from (2.2.5), $\dot{\phi} J_{1}=J_{2} \dot{\phi}$ if and only if

$$
J_{G}\left(\dot{L}_{\gamma(z)}(B)+\left(R_{\lambda} \dot{\circ} \gamma\right)_{z}(A)\right)+\dot{R}_{\gamma(z) \lambda} \eta(A)=\dot{L}_{\gamma(z)}\left(J_{G} B+\dot{R}_{\lambda} \omega(A)\right)+\left(R_{\lambda} \dot{\circ} \gamma\right)_{z}\left(J_{M} A\right) .
$$

Since left-translation is holomorphic and right and left translation commute we have $\xi$ is equivalent to $\psi$ if and only if there exists $\gamma: M \rightarrow G$ such that

$$
J_{G} \dot{\gamma}_{z}(A)+\dot{R}_{\gamma(z)} \eta(A)=\dot{L}_{\gamma(z)} \omega(A)+\dot{\gamma}_{z}\left(J_{M} A\right) .
$$

Proposition 2.2.6. In the above notation:

(1) If $\omega \sim_{\exp } \eta$ then $\xi$ is equivalent to $\psi$.

(2) If $G$ has the exponential lift property with respect to $M$ then $\omega \sim_{\exp } \eta$ if and only if $\xi$ is equivalent to $\psi$.

Proof. We shall prove (1) and (2) simultaneously. If $\omega \sim_{\exp } \eta$ then there is an $h: M \rightarrow \hat{G}$ satisfying equation (2.2.2). If $\gamma(z)=\exp (h(z))$ then we claim that $\gamma$ satisfies equation (2.2.4) of Lemma 2.2.5. If, on the other hand, $G$ has exponential lift with respect to $M$ and $\xi$ is equivalent to $\psi$ then we may find an $h: M \rightarrow \hat{G}$ and a $\gamma: M \rightarrow G$ such that $\gamma$ satisfies (2.2.4) of Lemma 2.2.5 and $\gamma=\exp \circ h$. In this case we must show that $h$ satisfies equation (2.2.2). These assertions are proven in the following computation: Let $\gamma(z)=\exp h(z)$ then for $X=h(z)$ equation (2.2.4) becomes

$$
J_{G} d(\exp )_{X}(d h(A))-d(\exp )_{X} d h\left(J_{M} A\right)=d L_{\exp X} \omega(A)-d R_{\exp X} \eta(A)
$$

or (because exp is holomorphic)

$$
d(\exp )_{X}\left(i d h(A)-d h\left(J_{M} A\right)\right)=d L_{\exp X} \omega(A)-d R_{\exp X} \eta(A)
$$

thus from equation (1.1.1) we have

$$
2 i d(\exp )_{X} \bar{\partial} h(A)=d L_{\exp X}(\omega(A))-d R_{\exp X}(\eta(A))
$$

but $\left[8\right.$, p. 95] $d(\exp )_{X}=d\left(L_{\exp X}\right)_{e} \circ\left(\left(1-e^{-\operatorname{ad} X}\right) / \operatorname{ad} X\right)$ so

$$
\begin{aligned}
2 i d\left(L_{\exp X}\right)_{e} \circ\left(\left(1-e^{-\operatorname{ad} X}\right) / \operatorname{ad} X\right) \bar{\partial} h(A) & =d\left(L_{\exp X}\right) \omega(A)-d R_{\exp X} \eta(A), \\
2 i\left(\left(I-e^{-\operatorname{ad} X}\right) / \operatorname{ad} X\right) \bar{\partial} h(A) & =\omega(A)-d\left(L_{\exp (-X)} \circ R_{\exp X}\right) \eta(A) .
\end{aligned}
$$

However $d\left(L_{\exp (-x)} \circ R_{\exp x}\right)=\operatorname{ad}(\exp X)$ which is known [5] to be $e^{\operatorname{ad} x}$; hence, after identifying $T(G)$ and $\hat{G}$, we obtain

$$
2 i\left(\left(I-e^{-\mathrm{ad} h(z)}\right) / \mathrm{ad} h(z)\right)(\bar{\partial} h(A))=\bar{\omega}(A)-e^{-\mathrm{ad} h(z)} \eta(A)
$$

which is equation (2.2.2).

COROllaRy 2.2.7. Let $M$ be a complex manifold, $G$ a complex Lie group. Let

$\Xi_{A}: A \exp D(M, \hat{G}) \rightarrow$ Equivalence classes of real product bundles

$$
[\omega] \leadsto J^{\omega}
$$


where $J^{\omega}$ is as in equation (2.2.3); then

(1) $\Xi_{A}$ is onto.

(2) If $G$ has the exponential lift property with respect to $M$ then $\Xi_{A}$ is an isomorphism.

The maps $\Xi$ and $\Xi_{A}$ are well defined by Proposition 2.2 .6 and onto by Corollary 1.2.2. Part (2) of Theorem 2.2.4 and Corollary 2.2.7 follow from part (2) of Proposition 2.2.6; hence all we need to prove our fundamental theorem is that $J^{\omega}$ is a complex structure if and only if $\omega$ is expclosed. To establish this fact we need the following:

LEMMA 2.2.8. Let $J$ be an almost complex structure on $M \times G$ coherent with the trivial bundle and let $V$ be an open set in $G$ containing the identity, then $J$ is a complex structure if and only if there is an open cover $\left\{U_{\alpha}\right\}$ of $M$ and maps $s_{\alpha}: U_{\alpha} \rightarrow V$ such that $\Sigma_{\alpha}: U_{\alpha} \rightarrow\left(U_{\alpha} \times G\right)_{J}$ given by $\Sigma_{\alpha}(u)=\left(u, s_{\alpha}(u)\right)$ is almost holomorphic for each $\alpha$.

Proof. Assume that $\Sigma_{\alpha}$ are given. Define $\Gamma_{\alpha}$ via

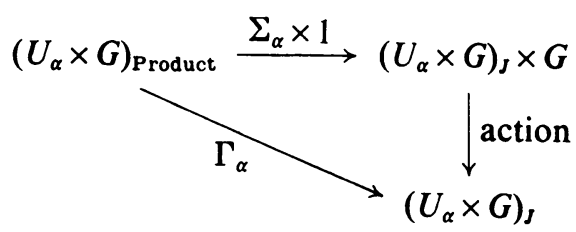

i.e. $\Gamma_{\alpha}(u, g)=\left(u, s_{\alpha}(u) g\right)$. Then $\Gamma_{\alpha}$ is an almost holomorphic diffeomorphism of $\left(U_{\alpha} \times G\right)_{\text {Product }}$ onto $\left(U_{\alpha} \times G\right)_{J}$ which says that $J$ is locally equivalent to the product structure. Because the product structure is a complex structure we have shown that $J$ is a complex structure.

If, on the other hand, $J$ is complex then there is an open cover $\left\{U_{\beta}^{\prime}\right\}$ of $M$ and local sections $\Sigma_{\beta}^{\prime}: U_{\beta}^{\prime} \rightarrow U_{\beta} \times G$ defined by $\Sigma_{\beta}^{\prime}(u)=\left(u, s_{\beta}^{\prime}(u)\right)$ which are $J$-holomorphic. Let $z$ be a given point in $M$. Choose any $U_{\beta}^{\prime}$ such that $z \in U_{\beta}^{\prime}$. Let $\alpha=(z, \beta)$ and $s_{\alpha}^{\prime}=L_{s_{\beta}^{\prime}(z)}^{-1} \circ s_{\beta}^{\prime}$. Let $U_{\alpha}=U_{\beta}^{\prime} \cap\left(s_{\alpha}^{\prime}\right)^{-1}(N)$ and $s_{\alpha}=s_{\alpha}^{\prime}$ restricted to $U_{\alpha}$; then $s_{\alpha}^{\prime}(z)$ $=L_{s_{\beta}^{\prime}(z)}^{-1} \circ s_{\beta}^{\prime}(z)=\left(s_{\beta}^{\prime}(z)\right)^{-1} s_{\beta}^{\prime}(z)=e \in V$, hence $z \in U_{\alpha}$. Clearly $s_{\alpha}$ is almost holomorphic as the restriction of the composition of almost holomorphic maps and $s_{\alpha}\left(U_{\alpha}\right)=s_{\alpha}\left(U_{\beta}^{\prime} \cap\left(s_{\alpha}^{\prime}\right)^{-1}(V)\right) \subset s_{\alpha}\left(s_{\alpha}^{\prime}\right)^{-1}(V)=V$.

The following proposition completes the proof of Theorem 2.2.4.

Proposition 2.2.9. Let $\omega \in \Lambda^{0,1}(M, \hat{G})$, then $\omega$ is expclosed if and only if $J^{\omega}$ is a complex structure.

Proof. If $\omega$ is expclosed then there is an open cover $\left\{U_{\alpha}\right\}$ of $M$ and $h_{\alpha}: U_{\alpha} \rightarrow \hat{G}$ such that equation (2.2.2) holds for each $\alpha$. Let $s_{\alpha}=\exp \circ h_{\alpha}$, then (from the proof of Proposition 2.2.6) $s_{\alpha}$ is almost holomorphic.

Conversely, suppose $J^{\omega}$ is a complex structure. It is well known that there is an open set $\tilde{V}$ of zero in $\hat{G}$ such that the restriction of the exponential map to $\tilde{V}$ is a 
diffeomorphism [5]. Let $V=\exp (\tilde{V})$ and apply Lemma 2.2 .8 to obtain $s_{\alpha}: U_{\alpha} \rightarrow V$ $\subseteq G$. Then $s_{\alpha}$ can be written as $s_{\alpha}(z)=\exp h_{\alpha}(z)$ for some $h_{\alpha}: U_{\alpha} \rightarrow \hat{G}$ (because $\exp ^{-1}$ exists on $V$ ). Since $s_{\alpha}$ gives an equivalence between $J$ and the product structure, then $h_{\alpha}$ yields the fact that $\omega$ restricted to $U_{\alpha}$ is expexact for each $\alpha$, hence $\omega$ is expclosed.

3. Computation of $\operatorname{Exp} D(M, G)$. In this section we shall apply the theory developed in the first two sections of this chapter. We shall first dispose of the abelian case by reproving Corollary 2.1 .3 and exhibiting the isomorphism between $\mathscr{D}_{0,1}(M, \hat{G})$ and Pic $(M, G)$ in a more concrete manner. We shall then examine the case in which $G$ is solvable. In [6], Frenkel (using the techniques of sheaf-theoretic cohomology) proves that if $G$ is solvable then the vanishing of $\mathscr{D}_{0,1}(M, C)$ implies the vanishing of $\operatorname{Pic}(M, G)$. We shall prove both a generalization of Frenkel's theorem and its converse (Theorems 2.3.2 and 2.3.4). We shall then calculate the exponentially closed $(0,1)$-forms for arbitrary $G$.

Let $\langle\eta\rangle$ be the $\bar{\partial}$-cohomology class of $\eta \in \Lambda^{0,1}(M, \hat{G})$.

THEOREM 2.3.1. Let $\pi_{1}(M)$ be a torsion group and $G$ be abelian, then the map $z: \mathscr{D}_{0,1}(M, \hat{G}) \rightarrow \operatorname{Pic}(M, G)$ given by $z(\langle\eta\rangle)=G \rightarrow(M \times G)_{J^{n}} \rightarrow M$ is an isomorphism.

Proof. By the fundamental theorem, it suffices to show that $\operatorname{Exp} D(M, G)$ $=\mathscr{D}_{0,1}(M, \hat{G})$ but this is exactly Proposition 2.2.3.

Let $h: M \rightarrow \hat{G}$, then for the remainder of this section we shall write

$$
A_{z}(h)=2 i \sum_{n=0}^{\infty} \frac{(-1)^{n}(\operatorname{ad} h(z))^{n}}{(n+1) !}=2 i \frac{e^{-\operatorname{ad} h(z)}-I}{\operatorname{ad} h(z)}
$$

for all $z \in M$. We shall also write $A(h)$ for $A_{z}(h)$ if there is no chance of confusion.

TheOREM 2.3.2. If $[\hat{G}, \hat{G}] \neq \hat{G}$ then $\operatorname{Exp} D(M, G)=0$ implies $\mathscr{D}_{0,1}(M, C)=0$.

Proof. Let $0 \neq e_{1} \in \hat{G}$ such that $e_{1} \notin[\hat{G}, \hat{G}]$. Let $\omega_{1}$ be a $\bar{\partial}$-cocycle. Let $\omega=\omega_{1} e_{1}$. We may find a locally defined function $h_{1}: U \rightarrow C$ such that $\omega_{1}=\bar{\partial} h_{1}$. Let $h=-i h_{1} e_{1} / 2 ;$ then

$$
A(h) \bar{\partial} h=2 i \bar{\partial} h+2 \sum_{n=1}^{\infty} \frac{(-1)^{n}(\operatorname{ad} h(u))^{n}}{(n+1) !} \bar{\partial} h=\bar{\partial} h_{1} e_{1}=\omega
$$

because $(\operatorname{ad} h) \bar{\partial} h=\left[h_{1} e_{1}, \bar{\partial} h_{1} e_{1}\right]=0 . \omega$ is therefore expclosed and hence by assumption expexact. Let $l: M \rightarrow \hat{G}$ be a function such that

$$
A(l) \bar{\partial} l=\omega .
$$

Let $\left\{e_{i}\right\}$ be any basis of $\hat{G}$ whose first element is $e_{1}$; then we may write $l=\sum l_{i} e_{i}$ where $l_{i}: M \rightarrow C$. Hence equation (2.3.1) becomes $\omega=A(l) \bar{\partial} l=\sum A(l)\left(\bar{\partial} l_{i}\right) e_{i}$ $=\left(2 i \bar{\partial} l_{1}\right) e_{1}+\alpha$ where $\alpha \in \hat{G}-C e_{1}$. Therefore $2 i \bar{\partial} l_{1}=\omega_{1}$ and so $\omega_{1}$ is a $\vec{\partial}$-coboundary. 
The above theorem is the converse of Frenkel's theorem. Before proving a generalization of Frenkel's theorem by our techniques, we shall need a lemma.

LEMMA 2.3.3. Let $\phi: \hat{G}_{1} \rightarrow \hat{G}_{2}$ be a Lie algebra homomorphism. If $\omega$ is expexact then so is $\phi(\omega)$.

Proof. Let $\omega \in \Lambda^{0,1}\left(M, \hat{G}_{1}\right)$ with $\omega=A(h) \bar{\partial} h$ where $h: M \rightarrow \hat{G}_{1}$. Let $l=\phi \circ h$ : $M \rightarrow \hat{G}_{2} ;$ then

$$
\begin{aligned}
\phi(\omega)(X) & =\phi(\omega(X))=\phi(A(h) \bar{\partial} h(X)) \\
& =\phi\left(2 i \sum_{n=0}^{\infty} \frac{(-1)^{n}(\operatorname{ad} h)^{n}}{(n+1) !} \bar{\partial} h(X)\right) \\
& =2 i \sum_{n=0}^{\infty} \frac{(-1)^{n}(\operatorname{ad} l)^{n}}{(n+1) !} \bar{\partial} l(X)=A(l) \bar{\partial} l(X) .
\end{aligned}
$$

THEOREM 2.3.4. If $G$ is solvable, then $\mathscr{D}_{0,1}(M, C)=0$ implies that $\operatorname{Exp} D(M, \hat{G})$ $=0$.

Proof. We proceed by induction on the dimension of $G$. We remark that the theorem clearly holds if $G$ is abelian (by Proposition 2.2.3), which enables us to start the induction. Let $Z$ be the center of the nil-radical of $\hat{G}$; then $Z$ is an abelian ideal of $\hat{G}$ with $\operatorname{dim} Z \geqq 1$. Let $V$ be any vector space such that $\hat{G}=V \oplus Z$ (where $\oplus$ is understood to be the direct sum as vector spaces, not necessarily as Lie algebras). Let $\pi: \hat{G} \rightarrow \hat{G} / Z$ be the canonical projection.

If $\omega$ is any expclosed $(0,1)$-form on $M$ with values in $\hat{G}$ then $\omega^{*}=\pi \omega$ is an expclosed $(0,1)$-form with values in $\hat{G} / Z$ by Lemma 2.3 .3 . By induction, we know that there is a $k^{*}: M \rightarrow G / Z$ such that $A\left(k^{*}\right) \bar{\partial} k^{*}=\omega^{*}$. Now, $\left.\pi\right|_{V^{*}}: V \rightarrow \hat{G} / Z$ is an isomorphism, hence there is a $k: M \rightarrow V$ such that $\pi k=k^{*}$. Let $\theta=A(k) \bar{\partial} k$, then $\omega-\theta$ is an expclosed $\hat{G}$-valued $(0,1)$-form over $M$. Because $\pi(\omega-\theta)=\pi \omega-\pi \theta=\omega^{*}$ $-\omega^{*}=0$ we have $\omega-\theta$ is, in fact, a $Z$-valued $(0,1)$-form over $M$, hence there is $l: M \rightarrow Z$ such that

$$
\omega-\theta=A(l) \bar{\partial} l .
$$

Now for any $Y \in Z$ we have $(\operatorname{ad} Y) \hat{G} \subset Z$, hence the map which ad $Y$ induces on $\hat{G} / Z$ is trivial; hence we may rewrite equation (2.3.2) as $\omega-e^{-\mathrm{ad} l} \theta=A(l) \bar{\partial} l$ and so $\omega \sim \sim_{\exp } \theta \sim_{\exp } 0$ which completes the proof.

We shall now give a simple criterion for $\omega \in \Lambda^{0,1}(M, \hat{G})$ to be expclosed. Recall that if $\alpha, \beta \in \Lambda^{1}(M, \hat{G})$ then $[\alpha, \beta]$ is defined to be an element of $\Lambda^{2}(M, \hat{G})$ via

$$
[\alpha, \beta](X, Y)=\frac{1}{2}([\alpha(X), \beta(Y)]-[\alpha(Y), \beta(X)]) \text { for all } X, Y \in T(M) \text {. }
$$

THEOREM 2.3.5. Let $\eta \in \Lambda^{0,1}(M, \hat{G})$ correspond to the almost complex structure $J^{\eta}$ on $M \times G$; then the following are equivalent:
(a) $\eta$ is expclosed,
(b) $J^{n}$ is a complex structure,
(c) $\bar{\partial} \eta=(i / 4)[\eta, \eta]$. 
We shall first prove a preliminary proposition. Let $\gamma \in \Lambda^{r}(M, \hat{G})$, define $\gamma^{\#} \in \Lambda^{r}(M \times G, \hat{G})$ via

$$
\gamma_{z, \lambda}^{\#}\left(\left(A_{1}, B_{1}\right),\left(A_{2}, B_{2}\right), \ldots,\left(A_{r}, B_{r}\right)\right)=\left(\operatorname{ad} \lambda^{-1}\right) \gamma_{z}\left(A_{1}, \ldots, A_{r}\right) .
$$

Let $\tau \in \Lambda^{1}(M \times G, \hat{G})$ be given by $\tau_{z, \lambda}(A, B)=L_{\lambda}-1 B$.

Proposition 2.3.6. For any $\gamma \in \Lambda^{1}(M, \hat{G}), d\left(\gamma^{\#}\right)=(d \gamma)^{\#}-\left[\tau, \gamma^{\#}\right]$.

Proof. For any $A_{1}, A_{2} \in T_{z}(M), B_{1}, B_{2} \in T_{\lambda}(G)$

$$
\begin{aligned}
2 d & \left(\gamma^{\#}\right)\left(\left(A_{1}, B_{1}\right),\left(A_{2}, B_{2}\right)\right) \\
& =\left(A_{1}, B_{1}\right) \text { ad } \lambda^{-1} \gamma\left(A_{2}\right)-\left(A_{2}, B_{2}\right)\left(\operatorname{ad} \lambda^{-1}\right) \gamma\left(A_{1}\right)-\operatorname{ad~} \lambda^{-1}\left[\gamma\left(A_{1}\right), \gamma\left(A_{2}\right)\right] \\
& =2(d \gamma)^{\#}+\left(0, B_{1}\right) \text { ad } \lambda^{-1} \gamma\left(A_{2}\right)-\left(0, B_{2}\right)\left(\operatorname{ad~} \lambda^{-1}\right) \gamma\left(A_{1}\right) .
\end{aligned}
$$

We must therefore prove

$$
\begin{aligned}
\frac{1}{2}\left\{\left(0, B_{1}\right) \text { ad } \lambda^{-1} \gamma\left(A_{2}\right)-\left(0, B_{2}\right) \text { ad } \lambda^{-1} \gamma\left(A_{1}\right)\right\} \\
=\frac{1}{2}\left\{\left[L_{\lambda^{-1}} B_{1}, \text { ad } \lambda^{-1} \gamma\left(A_{2}\right)\right]-\left[L_{\lambda^{-1}} B_{2}, \text { ad } \lambda^{-1} \gamma\left(A_{1}\right)\right]\right\} .
\end{aligned}
$$

It suffices to prove equation (2.3.3) when $B_{1}$ and $B_{2}$ are left-invariant vector fields. Let $B=B_{1}$ or $B_{2}$ and $A=A_{1}$ or $A_{2}$. Let $\alpha(t)=\lambda_{0} \exp (t B)$ and $f(\lambda)=\left(\operatorname{ad} \lambda^{-1}\right) \gamma(A)$.

$$
\begin{aligned}
\left(0, B_{1}\right) & \operatorname{ad} \lambda^{-1} \gamma(A)=\lim _{t \rightarrow 0} \frac{f(\alpha(t))-f\left(\lambda_{0}\right)}{t} \\
& =\lim _{t \rightarrow 0} \frac{\operatorname{ad}\left(\lambda_{0}^{-1} \exp (-t B)\right) \gamma(A)-\operatorname{ad} \lambda_{0}^{-1} \gamma(A)}{t}=\lim _{t \rightarrow 0} \frac{\left(\operatorname{ad} e^{-t B}-I\right) \operatorname{ad} \lambda_{0}^{-1} \gamma(A)}{t},
\end{aligned}
$$

hence

$$
\left(0, B_{1}\right) \text { ad } \lambda^{-1}(A)=-\left[L_{\lambda^{-1}} B \text {, ad } \lambda_{0}^{-1} \gamma(A)\right] .
$$

Substituting this last result into equation (2.3.3) yields the proposition.

We now proceed with the proof of Theorem 2.3.5. Firstly, note that the equivalence of (a) and (b) is exactly Proposition 2.2.9. Let $\omega$ be the element of $\Lambda^{0,1}(M \times G, \hat{G})$ which corresponds to $\eta \in \Lambda^{0,1}(M, \hat{G})$; then by the construction in Lemma 1.2.7 we have $\omega=\eta^{\#}$. By Theorem 1.2.3, we have that $J^{\eta}$ is complex if and only if

(2.3.4) $0=2 d \eta^{\#}\left(J^{\eta} X, Y\right)+2 d \eta^{\#}\left(X, J^{\eta} Y\right)+\left[\eta^{\#} X, \eta^{\#} Y\right]$ for all $X, Y \in T(M \times G)$. Let $X=(A, B), Y=(C, D)$, where $A, C \in T_{z}(M), B, D \in T_{\lambda}(G)$; then

$$
J_{z, \lambda}^{\eta}(X)=\left(J_{M} A, J_{G} B+d R_{\lambda} \eta(A)\right) \text { and } J_{z, \lambda}^{\eta}(Y)=\left(J_{M} C, J_{G} D+d R_{\lambda} \eta(C)\right) ;
$$

hence,

$$
2\left[\tau, \eta^{\#}\right]\left(J^{\eta} X, Y\right)=\left[\tau\left(J^{\eta} X\right), \eta^{\#} Y\right]-\left[\tau(Y), \eta^{\#}\left(J^{\eta} X\right)\right]
$$

hence,

$2\left[\tau, \eta^{\#}\right]\left(J^{\eta} X, Y\right)=\left[L_{\lambda^{-1}} J_{G} B+\operatorname{ad} \lambda^{-1} \eta(A), \operatorname{ad} \lambda^{-1} \eta(C)\right]-\left[L_{\lambda^{-1}} D, \operatorname{ad} \lambda^{-1} \eta\left(J_{M} A\right)\right] ;$ similarly,

$2\left[\tau, \eta^{\#}\right]\left(X, J^{\eta} Y\right)=\left[L_{\lambda^{-1}} B, \operatorname{ad} \lambda^{-1} \eta\left(J_{M} C\right)\right]-\left[L_{\lambda^{-1}} J_{G} D+\operatorname{ad} \lambda^{-1} \eta(C), \operatorname{ad} \lambda^{-1} \eta(A)\right]$. 
Adding yields

$$
\left[\tau, \eta^{\#}\right]\left(J^{\eta} X, Y\right)+\left[\tau, \eta^{\#}\right]\left(X, J^{\eta} Y\right)=\left[\eta^{\#}, \eta^{\#}\right](X, Y)
$$

because $\eta\left(J_{M} C\right)=-i \eta(C)$. Applying Proposition 2.3.6 to equation (2.3.4) we find: $J^{\eta}$ is complex $\Leftrightarrow$

$$
\begin{aligned}
0= & 2(d \eta)^{\#}\left(J^{\eta} X, Y\right)-2\left[\tau, \eta^{\#}\right]\left(J^{\eta} X, Y\right)+(d \eta)^{\#}\left(X, J^{\eta} Y\right) \\
& -2\left[\tau, \eta^{\#}\right]\left(X, J^{\eta} Y\right)+\left[\eta^{\#} X, \eta^{\#} Y\right]
\end{aligned}
$$

hence from equation (2.3.5)

$$
0=2\left\{(d \eta)^{\#}\left(J^{\eta} X, Y\right)+(d \eta)^{\#}\left(X, J^{\eta} Y\right)\right\}-\left[\eta^{\#} X, \eta^{\#} Y\right] .
$$

Thus $J^{\eta}$ is complex $\Leftrightarrow$ for all $A \in T_{z}(M), C \in T_{\lambda}(G)$

$$
d \eta\left(J_{M} A, C\right)+d \eta\left(A, J_{M} C\right)=\frac{1}{2}[\eta(A), \eta(C)] .
$$

We shall now show that equation (2.3.6) holds if and only if $\bar{\partial} \eta=(i / 4)[\eta, \eta]$ where $\bar{\partial}$ is, of course, taken with respect to the complex structure, $J_{M}$, of $M$.

Assume that equation (2.3.6) holds; then, since $(i / 4)[\eta, \eta]$ is of type $(0,2)$, we need only show that $(d \eta-(i / 4)[\eta, \eta])(A, C)=0$ for all $A, C \in T^{0,1}(M)$ because ( $J_{M}$ being complex) $d \eta$ has no $(2,0)$-component. Now if $A, C \in T^{0,1}(M)$ then equation (2.3.6) dictates

$$
\begin{aligned}
(i / 4)[\eta(A), \eta(C)] & =(i / 2)\left(d \eta\left(J_{M} A, C\right)+d \eta\left(A, J_{M} C\right)\right) \\
& =(i / 2)(-2 i d \eta(A, C))=d \eta(A, C),
\end{aligned}
$$

hence $d \eta-(i / 4)[\eta, \eta]$ is of type $(1,1)$ and so $\bar{\partial} \eta=(i / 4)[\eta, \eta]$.

Conversely, if $\bar{\partial} \eta=(i / 4)[\eta, \eta]$ then $(d \eta-(i / 4)[\eta, \eta])(A, C)=0$ if $A, C \in T^{0,1}(M)$. However,

$$
\begin{aligned}
d \eta\left(J_{M} A, C\right)+d \eta\left(A, J_{M} C\right) & =-2 i d \eta(A, C) \\
& =-2 i \bar{\partial}_{\eta}(A, C)=-\left(2 i^{2} / 4\right)[\eta, \eta]=\frac{1}{2}[\eta, \eta] .
\end{aligned}
$$

Thus $2 d \eta\left(J_{M} A, C\right)+2 d \eta\left(A, J_{M} C\right)=[\eta(A), \eta(C)]$ if $A, C \in T^{0,1}(M)$. If $A, C \in T^{1,0} M$ or $A \in T^{0,1} M$ and $C \in T^{1,0} M$ then both sides of the above equation are zero.

In the interests of completeness we shall now write down explicitly the equations governing $\omega \sim_{\exp } \eta$ if

$$
G=\left\{\left(\begin{array}{ccc}
1 & z_{1} & z_{3} \\
0 & 1 & z_{2} \\
0 & 0 & 1
\end{array}\right) \mid z_{i} \in C\right\}
$$

Let

$$
e_{1}=\left(\begin{array}{lll}
0 & 1 & 0 \\
0 & 0 & 0 \\
0 & 0 & 0
\end{array}\right), \quad e_{2}=\left(\begin{array}{lll}
0 & 0 & 0 \\
0 & 0 & 1 \\
0 & 0 & 0
\end{array}\right), \quad e_{3}=\left(\begin{array}{lll}
0 & 0 & 1 \\
0 & 0 & 0 \\
0 & 0 & 0
\end{array}\right)
$$


then we may write $\omega=\sum_{i=1}^{3} \omega_{i} e_{i}, \eta=\sum_{i=1}^{3} \eta_{i} e_{i}$ where $\omega_{i}, \eta_{i} \in \Lambda^{0,1}(M, C)$. A simple computation shows: $\omega \sim_{\exp } \eta \Leftrightarrow$ there are smooth functions $h_{i}: M \rightarrow C$ such that

$$
\begin{aligned}
\omega_{1}-\eta_{1} & =2 i \bar{\partial} h_{1}, \\
\omega_{2}-\eta_{2} & =2 i \bar{\partial} h_{2}, \\
\omega_{3}-\eta_{3}+h_{1} \eta_{2}-h_{2} \eta_{1} & =2 i \bar{\partial} h_{3}-i h_{1} \bar{\partial} h_{2}+i h_{2} \bar{\partial} h_{1} .
\end{aligned}
$$

Of course if $\mathscr{D}_{0,1}(M, C)=0$ then $\operatorname{Exp} D(M, G)=0$; however we can make the following amusing remark: if $\mathscr{D}_{0,1}(M, C) \neq 0$ then in any topology which $\operatorname{Exp} D(M, G)$ inherits from $\Lambda^{0,1}(M, \hat{G})$ it is not Hausdorff. Consider $\omega^{\lambda}=\lambda \alpha e_{1} ; \eta^{\lambda}=\lambda \alpha e_{1}+\alpha e_{3}$ where $\alpha$ is a $\bar{\partial}$-cocycle which is not a $\bar{\partial}$-coboundary. Clearly $\omega^{\lambda}$ and $\eta^{\lambda}$ are expclosed for all $\lambda$ by Theorem 2.3.5. Furthermore, if $\lambda \neq 0 h_{1}=0, h_{2}=1 / \lambda$ and $h_{3}=0$ satisfy equations (2.3.7) hence $\omega^{\lambda} \sim_{\exp } \eta^{\lambda}$ for $\lambda \neq 0$. However, $\lim _{\lambda \rightarrow 0} \omega^{\lambda}=0, \lim _{\lambda \rightarrow 0} \eta^{\lambda}=\alpha e_{3}$ and so $\lim _{\lambda \rightarrow 0} \omega^{\lambda}$ is not expcohomologous to $\lim _{\lambda \rightarrow 0} \eta^{\lambda}$. By passing to equivalence classes we see that we have a sequence in $\operatorname{Exp} D(M, \hat{G})$ which has 2 limits; hence $\operatorname{Exp} D(M, \hat{G})$ cannot be Hausdorff!

\section{Chapter III: Applications to Differential Geometry}

1. Applications to the theory of holomorphic connections. The following is immediate from Theorem 2, Theorem 5 and Proposition 12 of [1]:

THEOREM 3.1.1 (АTIYAH). A holomorphic principal fibre bundle with compact Kähler base whose structure group is $C^{*}$ possesses a holomorphic connection if and only if it is real trivial.

Atiyah's proof is algebraic. In this section we attempt to give a constructive proof of Atiyah's theorem if the structure group is abelian. We are successful in one direction but in the other direction we must assume that the compact Kähler base is simply connected. We also give a necessary and sufficient condition for a real trivial holomorphic fibre bundle with arbitrary structure group and base to admit a holomorphic connection (Corollary 3.1.6).

We begin by recalling some definitions referring to [1] for all details. Unless otherwise noted, we shall maintain the notation of the earlier chapters. Let $\xi: G \rightarrow P \stackrel{\pi}{\rightarrow} M$ be a holomorphic principal fibre bundle. Let $Q=T(P) / G$ and $L(P)=(\operatorname{Ker} \pi) / G$. Let $\lambda$ be the inclusion $L(P) \subset Q$.

$Q$ and $L(P)$ are both vector bundles over $M$ and $\mathscr{A}(\xi): 0 \rightarrow L(P) \stackrel{\lambda}{\rightarrow} Q \stackrel{\pi}{\rightarrow} T(M)$ $\rightarrow 0$ is an exact sequence of complex vector bundles called the fundamental sequence of $\xi$. A holomorphic connection for $\xi$ is a splitting of the exact sequence $\mathscr{A}(\xi)$; that is, a holomorphic map $\Gamma: Q \rightarrow L(P)$ such that $\Gamma \circ \lambda$ is the identity on $Q$. Recall that a connection form on $\xi: G \rightarrow P \rightarrow B$ is $\hat{G}$-valued 1 -form $\psi$ on $P$ such that

(i) $\psi_{p}\left(\theta_{p}(A)\right)=A$ for all $A \in \hat{G}$ and $p \in P$, and

(ii) $\left(R_{g}^{*} \psi\right)_{p}(X)=\left(\operatorname{ad} g^{-1}\right) \psi_{p}(X)$ for all $X \in T_{p}(P)$ and $p \in P$.

A connection is of type $(1,0)$ if $\psi(J X)=i \psi(X)$. 
Proposition 3.1.2. There is a holomorphic connection $\Gamma$ on $G \rightarrow P_{J} \rightarrow M$ if and only if there exists a connection form $\psi$ of type $(1,0)$ such that $\bar{\partial}_{J} \psi=0$.

Proof. Let $0 \rightarrow L(P) \stackrel{\lambda}{\rightarrow} Q(P) \stackrel{\pi}{\rightarrow} T(M) \rightarrow 0$ be the fundamental sequence for $\xi$. If $p \in P$ and $X \in T_{p}(P)$, let $[p, X]=$ image of $(p, X) \in T(P)$ in $Q(P)$ under the projection $T(P) \rightarrow T(P) / G=Q(P)$. If $\psi$ is a connection 1 -form then define $\Gamma([p, X])=\left\langle p, \theta_{p}(\psi(X))\right\rangle$ where $\theta_{p}: \hat{G} \rightarrow(\operatorname{Ker} \pi)_{p}$ is the usual isomorphism and $\langle p, Y\rangle$ denotes the equivalence class of $(p, Y) \in \operatorname{Ker} \pi$ in $L(P)$. $\Gamma$ is well defined because

$$
\begin{aligned}
\Gamma\left(\left[p^{g}, \dot{R}_{g} X\right]\right) & =\left\langle p^{g}, \theta_{p}\left(\psi\left(\dot{R}_{g} X\right)\right)\right\rangle=\left\langle p^{g}, \theta_{p^{g}}\left(\left(\operatorname{ad} g^{-1}\right) \psi(X)\right)\right\rangle \\
& =\left\langle p^{g}, R_{g} \theta_{p} \psi(X)\right\rangle=\left\langle p, \theta_{p}(\psi(X))\right\rangle .
\end{aligned}
$$

$\Gamma$ is then holomorphic as the composition of holomorphic maps. (We have used here that a $(1,0)$-form $P$ with values in $\hat{G}$ is holomorphic as a $(1,0)$-form if and only if it is holomorphic as a map of the complex manifold $T(P)$ to the complex manifold $\hat{G}$.) The converse follows from a similar construction.

Note that Proposition 3.1.2 says that $\psi$ is a holomorphic $(1,0)$-form on $P$.

Proposition 3.1.3. Let $\psi$ be a $\hat{G}$-valued connection 1-form on $(M \times G)_{J}$ where $J$ corresponds to $\omega \in \Lambda^{0,1}(M, \hat{G})$ via the fundamental theorem; then $\psi$ is of type $(1,0)$ with respect to $J$ if and only if there is $\beta \in \Lambda^{(1,0)}(M, \hat{G})$ such that

$$
\begin{aligned}
& \psi_{z, \lambda}(A, B)=L_{\lambda^{-1}} B+(i / 2)\left(\operatorname{ad} \lambda^{-1}\right) \pi^{*}((\beta-\omega)(A, B)) \\
& \qquad \text { for all } A \in T_{z}(M), B \in T_{\lambda}(G) .
\end{aligned}
$$

Proof. Clearly any form $\psi$ satisfying equation $(3.1 .1)$ is a $(1,0)$ connection. Assume now that $\psi$ is a connection and let $\psi_{z, \lambda}^{1}(A, B)=\psi_{z, \lambda}(A, 0)$; then from the second condition in the definition of a connection we have

$$
\begin{aligned}
\left(\operatorname{ad} \lambda^{-1}\right) \psi_{z, e}\left(A, \dot{R}_{\lambda}^{-1} B\right) & =\left(R_{\lambda}^{*} \psi\right)_{z, e}\left(A, \dot{R}_{\lambda}^{-1} B\right) \\
& =\psi_{z, \lambda}\left(A, \dot{R}_{\lambda} \dot{R}_{\lambda}^{-1} B\right)=\psi_{z, \lambda}(A, B) .
\end{aligned}
$$

But condition (1) of a connection demands $\psi_{z, e}\left(0, \dot{R}_{\lambda}-1 B\right)=\dot{R}_{\lambda}-1 B$; thus

$$
\psi_{z, \lambda}(A, B)=\left(\operatorname{ad} \lambda^{-1}\right)\left\{\psi_{z, e}(A, 0)+\psi_{z, e}\left(0, \dot{R}_{\lambda^{-1}} B\right)\right\},
$$

hence

$$
=\left(\operatorname{ad} \lambda^{-1}\right)\left(\psi_{z, e}^{1}(A)\right)+L_{\lambda}-1 B .
$$

$\psi_{z, e}^{1}(A)$ may be written $\pi^{*} \alpha$ for some $\alpha \in \Lambda^{1}(M, G)$ because $\left(\pi^{*} \alpha\right)_{z, \lambda}(A, B)$ is independent of both $B$ and $\lambda$.

$$
\begin{aligned}
\psi_{z, \lambda}(A, B) & =\left(\operatorname{ad} \lambda^{-1}\right) \pi^{*} \alpha(A, B)+L_{\lambda}-1 B, \\
\psi_{z, \lambda}(J(A, B)) & =\psi_{z, \lambda}\left(J_{M} A, J_{G} B+\dot{R}_{\lambda}(\omega(A))\right) ;
\end{aligned}
$$

hence

$$
\psi_{z, \lambda}(J(A, B))=L_{\lambda}-1\left(J_{G} B+\dot{R}_{\lambda} \omega(A)\right)+\left(\operatorname{ad} \lambda^{-1}\right) \alpha\left(J_{M} A\right)
$$


However

$$
i \psi_{z, \lambda}(A, B)=i L_{\lambda}-1 B+i\left(\operatorname{ad} \lambda^{-1}\right) \alpha(A) .
$$

Thus $\psi(J(A, B))=i \psi(A, B) \Leftrightarrow$

$$
i L_{\lambda^{-1}} B+i\left(\operatorname{ad} \lambda^{-1}\right)(\alpha(A))=L_{\lambda^{-1}} J_{G} B+\operatorname{ad} \lambda^{-1} \omega(A)+\left(\operatorname{ad} \lambda^{-1}\right)\left(\alpha\left(J_{M} A\right)\right)
$$

or $\psi(J(A, B))=i \psi(A, B) \Leftrightarrow i \alpha(A)=\omega(A)+\alpha\left(J_{M} A\right)$.

However for any $A \in T_{z}(M)$

$$
\begin{aligned}
\alpha(A) & =\left(i \alpha(A)-\alpha\left(J_{M} A\right)+i \alpha(A)+\alpha\left(J_{M} A\right)\right) / 2 i \\
& =-i \omega(A) / 2+\left(i \alpha(A)+\alpha\left(J_{M} A\right)\right) / 2 i,
\end{aligned}
$$

so

$$
\alpha(A)=-i \omega(A) / 2+i \beta(A) / 2
$$

where $\beta$ is an arbitrary element of $\Lambda^{(1,0)}(M, G)$. Substituting this last equation into equation (3.1.2) yields equation (3.1.1).

We shall now calculate the set of holomorphic connection forms on $G \rightarrow(M \times G)_{J}$ $\rightarrow M$. Recall that for any $\gamma \in \Lambda^{p}(M, \hat{G})$ we may define $\gamma^{\#} \in \Lambda^{p}(M \times G, \hat{G})$ by $\gamma_{z, \lambda}^{\#}=\left(\operatorname{ad~} \lambda^{-1}\right)\left(\pi^{*} \gamma\right)$ as in $\S 3$ of Chapter II. We also define $\tau: T(M \times G) \rightarrow \hat{G}$ by $\tau_{z, \lambda}(A, B)=L_{\lambda}-1 B$. Equation (3.1.1) now becomes (with $\psi_{0}=\tau-i \omega^{\sharp} / 2$ )

$$
\psi=\tau+(i / 2)(\beta-\omega)^{\#}=\psi_{0}+i \beta^{\#} / 2 .
$$

LEMMA 3.1.4. (a) $\partial \omega^{\#}=(\partial \omega)^{\#}-\left[\psi_{0}, \omega^{\#}\right]$.

(b) $\bar{\partial} \beta^{\#}=(\bar{\partial} \beta)^{\#-}-(i / 2)\left[\omega^{\#}, \beta^{\#}\right]$.

Proof. From Proposition 2.3.6 we have

and

$$
d \omega^{\#}=(d \omega)^{\#}-\left[\tau, \omega^{\#}\right]=(d \omega)^{\#}-\left[\psi_{0}, \omega^{\#}\right]-(i / 2)\left[\omega^{\#}, \omega^{\#}\right]
$$

$$
d \beta^{\#}=(d \beta)^{\#}-\left[\tau, \beta^{\#}\right]=(d \beta)^{\#}-\left[\psi_{0}, \beta^{\#}\right]-(i / 2)\left[\omega^{\#}, \beta^{\#}\right],
$$

hence the result follows by comparing type.

THEOREM 3.1.5. The set of holomorphic connections on $G \rightarrow(M \times G)_{J^{\omega}} \rightarrow M$ is in 1-1 correspondence with the set of all $\beta \in \Lambda^{1,0}(M, \hat{G})$ such that

$$
\bar{\partial} \beta=\partial \omega-(i / 2)[\omega, \beta] .
$$

Proof. We know from Proposition 3.1.3 that the set of $(1,0)$ connections is in 1-1 correspondence with the set of $\beta \in \Lambda^{1,0}(M, \hat{G})$ satisfying equation (3.1.1); hence we need only show that $\psi$ is holomorphic if and only if $\beta$ satisfies equation (3.1.3). We shall first calculate $\bar{\partial} \psi_{0}$. Since $d \tau=-\frac{1}{2}[\tau, \tau]$ we have

$$
\begin{aligned}
d \psi_{0} & =-\frac{1}{2}[\tau, \tau]-(i / 2) d \omega^{\#}=-\frac{1}{2}\left[\psi_{0}+(i / 2) \omega^{\#}, \psi_{0}+(i / 2) \omega^{\#}\right]-(i / 2) d \omega^{\#} \\
& =-\frac{1}{2}\left(\left[\psi_{0}, \psi_{0}\right]-\frac{1}{4}\left[\omega^{\#}, \omega^{\#}\right]+i\left[\psi_{0}, \omega^{\#}\right]-(i / 2) d \omega^{\#}\right)
\end{aligned}
$$

hence

$$
\bar{\partial} \psi_{0}=-(i / 2)\left[\psi_{0}, \omega^{\#}\right]-(i / 2) \partial \omega^{\#} ;
$$


note that $\beta^{\#} \in \Lambda^{1,0}(M \times G, \hat{G})$ hence $\bar{\partial} \psi=\bar{\partial} \psi_{0}+(i / 2) \bar{\partial} \beta^{\#}$, so $\bar{\partial} \psi=-(i / 2)\left[\psi_{0}, \omega^{\#}\right]$ $-(i / 2) \partial \omega^{\#}+(i / 2) \bar{\partial} \beta^{\#}$. Applying Lemma 3.1.4,

$$
\bar{\partial} \psi=-(i / 2)\left[\psi_{0}, \omega^{\#}\right]-(i / 2)\left((\partial \omega)^{\#}-\left[\psi_{0}, \omega^{\#}\right]\right)+(i / 2)\left((\bar{\partial} \beta)^{\#}-(i / 2)\left[\omega^{\#}, \beta^{\#}\right]\right) ;
$$

hence

$$
\bar{\partial} \psi=(i / 2)(\bar{\partial} \beta-\partial \omega-(i / 2)[\omega, \beta]) \# .
$$

COROLlaRY 3.1.6. $\xi: G \rightarrow(M \times G)_{J^{\omega}} \rightarrow M$ admits a holomorphic connection if and only if there is $a \beta \in \Lambda^{1,0}(M, G)$ such that $\bar{\partial} \beta=\partial \omega-(i / 2)[\omega, \beta]$.

THEOREM 3.1.7. If $M$ is a compact Kähler manifold then any real trivial holomorphic principal fibre bundle over $M$ with abelian structure group admits a holomorphic connection.

Proof. By Corollary 3.1.6 it suffices to produce $\beta \in \Lambda^{1,0}(M, \hat{G})$ such that $\bar{\partial} \beta=\partial \omega$.

We shall use the machinery of harmonic forms. Let $\bar{\partial}^{t}$ (resp. $d^{t}$ ) be the transpose of $\bar{\partial}$ (resp. $d$ ) with respect to the Hodge inner product on $\Lambda^{p}(M, \hat{G})$. Let $H(\eta)$ denote the harmonic part of $\eta \in \Lambda^{p}(M, \hat{G})$ and let $\Delta=2\left(\bar{\partial} \bar{\partial}^{t}+\bar{\partial}^{t} \bar{\partial}\right)$ be the Laplacian and $G$ be the Green's operator. Since the space of harmonic forms is $\left(\operatorname{Ker} d \cap \operatorname{Ker} d^{t}\right)$, we have that $\partial H(\gamma)=0$ for all $\alpha$. We have the decomposition $\omega=H(\omega)+G \Delta \omega$ or $\omega=H(\omega)+2 G\left(\bar{\partial}^{t}+\bar{\partial}^{t} \bar{\partial}\right) \omega$; hence $\partial \omega=-2 \bar{\partial} G \partial \bar{\partial}^{t} \omega-2 G \bar{\partial}^{t} \partial \bar{\partial} \omega$ or (since $J^{\omega}$ is complex, $\bar{\partial} \omega=0$ )

$$
\partial \omega=\bar{\partial} \beta \quad \text { if } \beta=-2 G \partial \bar{\partial}^{t} \omega .
$$

Note that the above proof is constructive; the connection defined above is, for $A \in T_{2}(M), B \in T_{\lambda}(G)$,

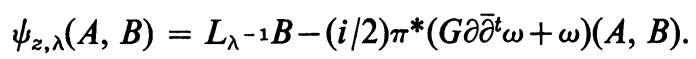

We now examine the converse of Theorem 3.1.7 in the abelian case.

THEOREM 3.1.8. Let $\xi: G \rightarrow P \rightarrow M$ be a holomorphic principal fibre bundle with $G$ abelian and $M$ compact Kähler. If $\xi$ has a holomorphic connection then $\xi$ has a flat connection.

Proof. Let $\omega \in \Lambda^{0,1}(M, \hat{G})$ corresponding to $\xi$ via Proposition 1.2.4. We shall use the notation of the proof of Theorem 3.1.7. Let $\alpha_{1}=-2 \partial \bar{\partial}^{t} G \omega$ then equation (3.1.5) yields $\bar{\partial} \alpha_{1}=\partial \omega$ and $\partial \alpha_{1}=0$. If $\psi$ is a holomorphic connection on $\xi$ then $\psi=\pi^{*} \gamma$ for some $\gamma \in \Lambda^{1,0}(M, \hat{G})$ such that $\bar{\partial} \gamma=0$. Let $\alpha_{2}=2 \delta^{t} \delta G \gamma$; then

$$
\begin{aligned}
\partial \alpha_{2} & =2 \partial \partial^{t} \partial G \gamma=2\left(\partial \partial^{t} \partial G \gamma+\partial \partial \partial^{t} G \gamma\right)+\partial H(\gamma) \\
& =\partial(\Delta G \gamma+H(\gamma))=\partial \gamma
\end{aligned}
$$

and

$$
\bar{\partial} \alpha_{2}=2 \partial^{t} \partial G \bar{\partial} \gamma=0
$$


Let $\beta=\alpha_{1}+(2 / i) \alpha_{2} \in \Lambda^{1,0}(M, G)$, then

$$
\partial \beta=(2 / i) \partial \alpha_{2}=(2 / i) \partial \gamma ;
$$

hence

(3.1.7) $(i / 2) \pi^{*} \partial \beta=\partial \psi$,

(3.1.8) $\bar{\partial} \beta=\bar{\partial} \alpha_{1}=\partial \omega$.

Now, let $\tilde{\psi}=\psi+(i / 2) \pi^{*}(\omega-\beta)$, then if $\tilde{\Omega}$ is the curvature of $\tilde{\psi}$ we have $d \tilde{\psi}=-\frac{1}{2}[\tilde{\psi}, \tilde{\psi}]+\tilde{\Omega}=\tilde{\Omega}$, hence $\widetilde{\Omega}^{1,1}=\bar{\partial} \psi+(i / 2) \pi^{*}(\partial \omega-\bar{\partial} \beta)=0$ because equation (3.1.8) says $\partial \omega=\bar{\partial} \beta$. Also

$$
\tilde{\Omega}^{2,0}=\partial \psi+(i / 2) \pi^{*}(\bar{\partial} \omega-\partial \beta)=\partial \psi+(i / 2) \pi^{*} \partial \beta=0
$$

where the last equality is equation (3.1.7). We therefore see that $\tilde{\psi}$ is a flat connection.

COROLlARY 3.1.9. Let $M$ be a simply connected compact Kähler manifold and $G$ be an abelian complex Lie group. Then if $\xi: G \rightarrow P \rightarrow M$ is a holomorphic principal fibre bundle which admits a holomorphic connection, then $\xi$ is a real trivial holomorphic principal fibre bundle.

Proof. This result is immediate from [9, p. 92].

2. Examples of Kähler manifolds. Given the manifold $P_{J}=(M \times G)_{J}$ where $J$ corresponds to $\eta \in \Lambda^{0,1}(M, \hat{G})$ as in Theorem 1.2.2 we shall now put a natural Hermitian metric on $P_{J}$ and obtain a necessary and sufficient condition in terms of $\eta$ for $P_{J}$ to be Kähler with respect to this metric. Recall that a Riemannian metric on $P$ is a smooth inner product on the tangent space of $P$ at each point. An (almost) Hermitian metric on the (almost) complex manifold $P_{J}$ is a Riemannian metric on $P$ such that $g(J X, J Y)=g(X, Y)$ for all $X, Y \in T(M)$ where $J$ is the (almost) complex structure on $P$. Let $\Omega(X, Y)=g(X, J Y)$ for $X, Y \in T(M)$, then $\Omega$ is a 2-form on $M$, called the Kähler form of $g$. If $d \Omega=0$ then the (almost) Hermitian metric is said to be (almost) Kähler.

PROPOSITION 2.4.1. Let $\xi_{0}: G \rightarrow P_{J_{0}} \rightarrow M$ be an almost holomorphic principal fibre bundle and let $J$ be an almost complex structure on $P$ coherent with $\xi_{0}$. Then $g$ is a Hermitian metric on $P$ where $g$ is defined by

$$
\begin{aligned}
g_{p}(X, Y)= & 2 g_{0}(X, Y)+g_{0}\left(J_{0} X, \theta_{p}(\omega(Y))\right) \\
& +g_{0}\left(J_{0} Y, \theta_{p}(\omega(X))\right)+g_{0}\left(\theta_{p}(\omega(X)), \theta_{p}(\omega(Y))\right)
\end{aligned}
$$

where $g_{0}$ is any metric on $P$ which is Hermitian with respect to $J_{0}, J$ corresponds to $\omega$ via Theorem 1.2.1 and $\theta_{p}$ is the usual embedding $\hat{G} \rightarrow(\operatorname{Ker} \pi)_{p}$.

Proof. Let $\tilde{g}(X, Y)=g_{0}(X, Y)+g_{0}(J X, J Y)$, then since

$$
\tilde{g}(J X, J Y)=g_{0}(J X, J Y)+g_{0}(X, Y)=\tilde{g}(X, Y),
$$


$\tilde{\mathbf{g}}$ is Hermitian with respect to $J$. The correspondence of Theorem 1.2.1 is given by $J_{p}(X)=\left(J_{0}\right)_{p}(X)+\theta_{p}\left(\omega_{p}(X)\right)$ for $p \in P$. Hence

$$
\begin{aligned}
\tilde{g}(X, Y)= & g_{0}(X, Y)+g_{0}\left(J_{0} X+\theta(\omega(X)), J_{0} Y+\theta(\omega(Y))\right) \\
= & g_{0}(X, Y)+g_{0}\left(J_{0} X, J_{0} Y\right) \\
& +g_{0}\left(J_{0} X, \theta(\omega(Y))+g_{0}\left(\theta(\omega(X)), J_{0} Y\right)+g_{0}(\theta(\omega(X)), \theta(\omega(Y)))\right.
\end{aligned}
$$

and so the result follows because $g_{0}$ is Hermitian with respect to $J_{0}$.

We shall now calculate the fundamental form of $g$. We shall write $\Omega^{\circ}(X, Y)$ $=g_{0}\left(X, J_{0} Y\right)$.

$$
\begin{aligned}
\Omega_{J}(X, Y)= & 2 g_{0}(X, J Y)+g_{0}\left(J_{0} X, \theta(\omega(J Y))\right) \\
& +g_{0}\left(J_{0} J Y, \theta(\omega(X))\right)+g_{0}(\theta(\omega(X)), \theta(\omega(J Y))) \\
= & 2\left\{\Omega^{0}(X, Y)+g_{0}(X, \theta(\omega(Y)))\right\}+g_{0}\left(J_{0} X, \theta\left(\omega\left(J_{0} Y\right)+\theta(\omega(Y))\right)\right) \\
& +g_{0}\left(J_{0}^{2} Y+J_{0} \theta(\omega(Y)), \theta(\omega(X))\right)+g_{0}\left(\theta(\omega(X)), \theta\left(\omega\left(J_{0} Y\right)\right)+\theta(\omega(Y))\right) .
\end{aligned}
$$

However since $\omega(\operatorname{Ker} \pi)=0$ we obtain

$$
\begin{aligned}
\Omega_{\jmath}(X, Y)= & 2 \Omega^{\circ}(X, Y)+2 g_{0}(X, \theta(\omega(Y)))+g_{0}\left(J_{0} X, \theta\left(\omega\left(J_{0} Y\right)\right)\right) \\
& -g_{0}(Y, \theta(\omega(X)))+g_{0}\left(J_{0} \theta(\omega(Y)), \theta(\omega(X))\right) \\
& +g_{0}\left(\theta(\omega(X)), \theta\left(\omega\left(J_{0} Y\right)\right)\right) .
\end{aligned}
$$

Since $\omega\left(J_{0} X\right)=-i \omega(X)$ and $\theta(i A)=J_{0} \theta(A)$,

$$
\begin{aligned}
\Omega_{J}(X, Y)= & 2 \Omega^{0}(X, Y)+2 g_{0}(X, \theta(\omega(Y)))-g_{0}\left(J_{0} X, J_{0} \theta(\omega(Y))\right) \\
& -g_{0}(Y, \theta(\omega(X)))+g_{0}\left(J_{0} \theta(\omega(Y)), \theta(\omega(X))\right) \\
& -g_{0}\left(\theta(\omega(X)), J_{0} \theta(\omega(Y))\right),
\end{aligned}
$$

and so we have proved

Proposition 2.4.2. Let $J$ be an almost complex structure on $P$ coherent with $\xi_{0}$; then the Kähler form of $P_{J}$ in the metric of Proposition 2.4.1 is

$$
\Omega_{\jmath}(X, Y)=2 \Omega^{0}(X, Y)+g_{0}(X, \theta(\omega(Y)))-g_{0}(Y, \theta(\omega(X))) .
$$

If the $g^{0}$ metric on $P_{J_{0}}$ is Kähler we still cannot expect a priori that the Hermitian metric $g$ (with respect to $J$ ) on $P$ will be Kähler. The Hopf-Calabi-Eckmann manifolds furnish examples of bundles in which the base (a product of projective spaces) and fibre (a torus) are Kähler but the total spaces do not even possess a Kähler metric (see [10] for details).

We shall now specialize the results of Proposition 2.4.2 to the real product case. Let $\eta$ be a $\hat{G}$-valued 1-form on $M$ such that $\eta\left(J_{0} X\right)=i \eta(X)$. We define the Kähler defect 2-form of $\eta, \Gamma^{\eta}$, by for $z \in M, \lambda \in G, A, A^{\prime} \in T_{z}(M), B, B^{1} \in T_{\lambda}(G)$,

$$
\Gamma_{z, \lambda}^{\eta}\left((A, B),\left(A^{\prime}, B\right)\right)=g_{G}\left(B,\left(d R_{\lambda}\right)_{e} \eta\left(A^{\prime}\right)\right)-g_{G}\left(B^{\prime},\left(d R_{\lambda}\right)_{e} \eta(A)\right)
$$

where $g_{G}$ is a right invariant Hermitian metric on $G$. 
TheOrem 2.4.3. Let $g_{M}$ be a Hermitian metric on $M, g_{G}$ a right invariant Hermitian metric on $G$. Let

$$
\begin{aligned}
g_{z, \lambda}\left((A, B),\left(A^{\prime}, B^{\prime}\right)\right)= & 2\left\{g_{M}\left(A, A^{\prime}\right)+g_{G}\left(B, B^{\prime}\right)\right\}+g_{G}\left(i B,\left(d R_{\lambda}\right)_{e} \eta\left(A^{\prime}\right)\right) \\
& +g_{G}\left(i B^{\prime},\left(d R_{\lambda}\right)_{e} \eta(A)\right)+g_{G}(\eta(A), \eta(B))
\end{aligned}
$$

and let $J=J^{\eta}$ be an almost complex structure on $M \times G$ as in Theorem 1.2.6; then $g$ is a Hermitian metric on $(M \times G)_{J}$ which is Kähler if and only if $d \Gamma^{n}=0$.

Proof. Let $g_{0}$ be the product Riemannian metric on $M \times G\left(\right.$ i.e. $g_{0}\left((A, B),\left(A^{\prime}, B\right)\right)$ $\left.=g_{M}\left(A, A^{\prime}\right)+g_{G}\left(B, B^{\prime}\right)\right)$. If $p=(z, \lambda)$ then $\theta_{p}(C)=\left(0,\left(d R_{\lambda}\right)_{e}(C)\right)$ for all $C \in \hat{G}$ and so $g$ is Hermitian by Proposition 2.4.1. However, if $X=(A, B), Y=\left(A^{\prime}, B^{\prime}\right)$ then

$$
g_{0}(X, \theta(\omega(Y)))=g_{0}\left((A, B),\left(0, d R_{\lambda}\left(\eta\left(A^{\prime}\right)\right)\right)\right)=g_{G}\left(B, d R_{\lambda}\left(\eta\left(A^{\prime}\right)\right)\right),
$$

hence

$$
\begin{aligned}
g_{0}(X, \theta \omega(Y))-g_{0}(Y, \theta \omega(X)) & =g_{G}\left(B, d R_{\lambda}\left(\eta\left(A^{\prime}\right)\right)\right)-g_{G}\left(B^{\prime}, d R_{\lambda}(\eta(A))\right) \\
& =\Gamma_{z, \lambda}\left((A, B),\left(A^{\prime}, B^{\prime}\right)\right) .
\end{aligned}
$$

We shall now compute the Kähler defect of $\eta \in \Lambda^{1}(M, \hat{G})$ if $G$ is a complex torus. We may then identify $\hat{G}$ with $C^{r}$ and $R_{\lambda}: G \rightarrow G$ is given by $R_{\lambda}(g)=g+\lambda$ for $g, \lambda \in G$, hence $\left(d R_{\lambda}\right)_{e}$ is the identity for any $\lambda \in G$. Let $\eta=\sum_{i=1}^{2 r} \eta_{i} e_{i}$ where $\left\{e_{1}, \ldots, e_{r}\right\}$ is the usual basis for $R^{2 r}=C^{r}$. Locally we have the coordinate chart $\left(x^{1}, \ldots, x^{n}, y^{\prime}, \ldots, y^{n}\right)$. Let $m^{i}=x^{i}, i \leqq n, m^{i+n}=y^{i}$, for $i>n$; then $\left\{d m^{i}\right\}_{i=1}^{2 n}$ form a local basis for $\Lambda^{1}(M),\left\{\delta / \delta m^{i}\right\}$ form a local basis for $T(M)$. Let $\left\{d g^{i}\right\}_{i=1}^{2 r}$ and $\left\{\delta / \delta g^{i}\right\}$ be defined similarly for $G$. We may then write

$$
\begin{aligned}
\Gamma(z, \lambda) & =\sum_{i, j}\left\{\Gamma_{i j} d m^{i} \wedge d m^{j}+\Gamma_{i, r+j} d m^{i} \wedge d g^{j}+\Gamma_{n+i, r+j} d g^{i} \wedge d g^{j}\right\}, \\
\Gamma_{i j}(z, \lambda) & =\Gamma_{z, \lambda}\left(\frac{\delta}{\delta m^{i}}, \frac{\delta}{\delta m^{j}}\right)=g\left(0, \eta\left(\frac{\delta}{\delta m^{j}}\right)\right)-g\left(0, \eta\left(\frac{\delta}{\delta m^{i}}\right)\right)=0 ;
\end{aligned}
$$

hence $\Gamma_{i j}=0$ for $1 \leqq i, j \leqq n$. Similarly $\Gamma_{n+i, r+j}=0$.

$$
\begin{aligned}
\Gamma_{i, n+j}(z, \lambda) & =\Gamma_{z, \lambda}\left(\frac{\delta}{\delta m^{i}}, \frac{\delta}{\delta g^{j}}\right)=g(0, \eta(0))-g\left(\frac{\delta}{\delta g^{j}}, \eta\left(\frac{\delta}{\delta m^{i}}\right)\right) \\
& =-g\left(\frac{\delta}{\delta g^{j}}, \sum_{k} \eta_{k}\left(\frac{\delta}{\delta m^{i}}\right) e_{k}\right)=\sum_{k} g_{j k} \eta_{k}\left(\frac{\delta}{\delta m^{i}}\right)=-\eta_{j}\left(\frac{\delta}{\delta m^{i}}\right)=-\eta_{j i}
\end{aligned}
$$

where $\eta_{k}=\sum_{l} \eta_{k l} d m^{l}$, so

$$
\Gamma(z, \lambda)=-\sum_{i, j} \eta_{j i}(z) d m^{i} \wedge d g^{j}=-\sum_{j} \eta_{j} \wedge d g^{j} .
$$

Observing that $\Gamma$ is independent of $\lambda \in G$, we have $d \Gamma(z, \lambda)=-\sum_{j} d \eta_{j} \wedge d g^{j}$, hence

THEOREM 2.4.4. Let $G$ be any complex torus, then the following are equivalent:

(a) $(M \times G)_{J^{n}}$ is Kähler in the metric of Theorem 2.4.3.

(b) $(M \times G)_{J^{n}}$ is almost Kähler in the metric of Theorem 2.4.4.

(c) $d \eta=0$. 
Added in proof. The author now has an example of a real product bundle with compact base and abelian structure group which does not admit a holomorphic connection, showing that the assumption of Kähler base is necessary in Theorem 3.1.7.

\section{BIBLIOGRAPHY}

1. M. F. Atiyah, Complex analytic connections in fibre bundles, Trans. Amer. Math. Soc. 85 (1957), 181-207. MR 19, 172.

2. L. Auslander, L. Green and F. Hahn, Flows on homogeneous spaces, Ann. of Math. Studies, no. 53, Princeton Univ. Press, Princeton, N. J., 1963. MR 29 \#4841.

3. H. Cartan, Problèmes globaux dans la théorie des fonctions analytiques de plusieurs variables complexes, Proc. Internat. Congress of Mathematicians (Cambridge, Mass., 1950), vol. 1, Amer. Math. Soc., Providence, R. I., 1952, pp. 152-164. MR 13, 548.

4. S. S. Chern, Complex manifolds without potential theory, Van Nostrand Math. Studies, no. 15, Van Nostrand, Princeton, N. J., 1967. MR 37 \#940.

5. C. Chevalley, Theory of Lie groups. I, Princeton Math. Series, vol. 8, Princeton Univ. Press, Princeton, N. J., 1946. MR 7, 412.

6. J. Frenkel, Cohomologie non abélienne et espaces fibrés, Bull. Soc. Math. France 85 (1957), 135-220. MR 20 \#4662.

7. R. Gunning, Lectures on Riemann surfaces, Princeton Math. Notes, Princeton Univ. Press, Princeton, N. J., 1966. MR 34 \#7789.

8. S. Helgason, Differential geometry and symmetric spaces, Pure and Appl. Math., vol. 12, Academic Press, New York, 1962. MR 26 \#2986.

9. S. Kobayashi and K. Nomizu, Foundations of differential geometry. Vol. I, Interscience, New York, 1963. MR 27 \#2945.

10. - Foundations of differential geometry. Vol. II, Interscience Tracts in Pure and Appl. Math., no. 15, vol. 2, Interscience, New York, 1969. MR 38 \#6501.

11. Séminaire de topologie algébrique de l'École Normale Supérieure, 1950-1951, Exposés 14-20 par H. Cartan. MR 14, 670.

12. E. Spanier, Algebraic topology, McGraw-Hill, New York, 1966. MR 35 \#1007.

13. N. E. Steenrod, The topology of fibre bundles, Princeton Math. Series, vol. 14, Princeton Univ. Press, Princeton, N. J., 1951. MR 12, 522.

Department of Mathematics, Southern Illinois University, Carbondale, Illinois 62901 